\title{
Modulation of Astrocytes on Mode Selection of Neuron Firing Driven by Electromagnetic Induction
}

\author{
Zhongquan Gao, ${ }^{1}$ Zhixuan Yuan, ${ }^{2}$ Zuo Wang, ${ }^{1}$ and Peihua Feng $\mathbb{D}^{2}$ \\ ${ }^{1}$ School of Power and Energy Engineering, Xi'an Jiaotong University, Xi'an, Shaanxi 710049, China \\ ${ }^{2}$ State Key Laboratory for Strength and Vibration of Mechanical Structures, School of Aerospace Engineering, \\ Xi'an Jiaotong University, Xi'an 710049, China \\ Correspondence should be addressed to Peihua Feng; fphd2017@xjtu.edu.cn
}

Received 5 September 2020; Revised 7 November 2020; Accepted 20 November 2020; Published 1 December 2020

Academic Editor: Jianzhong Su

Copyright ( 92020 Zhongquan Gao et al. This is an open access article distributed under the Creative Commons Attribution License, which permits unrestricted use, distribution, and reproduction in any medium, provided the original work is properly cited.

Both of astrocytes and electromagnetic induction are magnificent to modulate neuron firing by introducing feedback currents to membrane potential. An improved astro-neuron model considering both of the two factors is employed to investigate their different roles in modulation. The mixing mode, defined by combination of period bursting and depolarization blockage, characterizes the effect of astrocytes. Mixing mode and period bursting alternatively appear in parameter space with respect to the amplitude of feedback current on neuron from astrocyte modulation. However, magnetic flux obviously plays a role of neuron firing inhibition. It not only repels the mixing mode but also suppresses period bursting. The mixing mode becomes period bursting mode and even resting state when astrocytes are hyperexcitable. Abnormal activities of astrocytes are capable to induce depolarization blockage to compose the mixing mode together with bursting mode. But electromagnetic induction shows its strong ability of inhibition of neuron firing, which is also illustrated in the bifurcation diagram. Indeed, the combination of the two factors and appropriate choice of parameters show the great potential to control disorder of neuron firing like epilepsy.

\section{Introduction}

In order to understand complex physical and biochemical processes of signals generation and propagating in neural system, a powerful and basic model is proposed by Hodgkin and Huxley from a large number of experimental data on surface membrane of giant nerve of squids [1]. The Hodgkin-Huxley (HH) model explains successfully the switch between resting state and action potential from perspective of activation and inactivation of ion channels distributed in membrane of neuron. Different types of neuron firing like spiking and bursting can be captured by $\mathrm{HH}$ model, and it allows chaotic firing of neurons [2]. There are four variables in $\mathrm{HH}$ model, based on which, several simplified versions of model are developed to capture the main dynamics of neuron firing [3, 4]. Among them, ML (Morris and Lecar) model with three variables is considered to be elegant and simple to reveal dynamics of neuron firing [5].

Hundreds of studies have shown the validity and universality of ML model. Tsumoto proofs that the neuron firing can be adjusted by a single parameter via bifurcation theory [6]. The model can describe the oscillatory dynamics as a pacemaker triggered by Hopf bifurcation in muscle cells [7]. Ciszak distinguishes waking states and sleep states from perspective of neuron firing by ML model coupled with the spike time-dependent plasticity rule (STDP) [8]. Besides individual neuron firing activity, many researchers prefer to employ ML model to reveal dynamics of neuron spatiotemporal pattern in regular of complex neuronal networks. $\mathrm{Hu}$ proposes a complete electronic implementation of ML model for experimentally studying collective behaviors of neuronal network [9]. Wang discovers transition of pattern in a regular network consisting ML neurons coupled by chemical synapses [10]. And Feng further reveals the temporal property of pattern formation, which is characterized by phenomenon of critical slowing down [11].

Mode selection of neuron electrical activity is adjusted by different types of factors. It is believed that these factors, including ion channel noise [12], body temperature [13] and time delay $[14,15]$, are considered to trigger or suppress 
mental disorder via transferring neuron firing modes. More importantly, besides these factors, neuron firing is essentially modulated by glial cells. It is proofed experimentally that astrocytes not only support and nourish neurons but also involve in adjusting neuron information propagating among neuronal network through synapses [16-18]. Researchers illustrate the importance of calcium, glutamate, and ATP in chemical process of signal modulation between neurons by astrocytes. The glutamate, released from neurons to synaptic cleft, partially bounds to the receptors (mGluR) of the astrocytes membrane, leading generation of inositol 1,4,5-triphosphate (IP3) as the second messenger to trigger the release of calcium from endoplasmic reticulum. Stimulated by $\mathrm{Ca} 2+$ concentration increase, astrocytes release the glutamate to induce an additional inward current of neuron to influence information process. Suhita et al. propose a neuro-glial communication model based on $\mathrm{HH}$ model and predict spontaneous oscillations of neuron firing achieved by astrocyte modulation only [19]. Furthermore, astrocyte-neuron interaction is introduced into ML model to explore functionalbased procedure with coexistence of excitatory and inhibitory synapses [20].

Furthermore, signal propagation in cortex achieved by electrical activity of neuron firing is affected greatly by electromagnetic environment inside skull according to Maxwell electromagnetic induction theorem [21]. It is hard to describe coupling of neuron firing and electromagnetic induction and radiation until invention of memristor [22, 23], which bridges neuron activity and electromagnetic flux via an equivalent current. Indeed, electromagnetic induction regulates neuron firing both in scale of individual neuron activity and in scale of spatiotemporal pattern formation of neuronal network. A model is established to study mode selection, and a practical circuit is designed to simulate neuron firing for this improved model [24]. Firing mode transition is achieved by magnetic flux [25], and it is further characterized as a route to chaos of neuron firing via frequent alternation of periodic and quasiperiodic motion [26, 27]. Parastesh et al. introduce discontinuous magnetic induction into neuron model [28]. Yuan numerically study the delay effect of external stimulation with consideration of magnetic flux [29]. Besides, many studies focus on collective behaviors in neuron network and pattern formation and stability loss process controlled by electromagnetic field effects [30]. In particular, Tian et al. consider magnetic flux in a chainshape network of neurons and discovery the chimera state, a coexistence of synchronization and disorder state [31].

However, a comprehensive impact to neuron firing adjusted by astrocytes and exposed to electromagnetic environment does not explored deeply. In this paper, we address this problem by a neuro-glial system based on the ML model with consideration of electromagnetic induction.

Materials are listed as follows: a neuro-glial model modulated by electromagnetic field is described in Section 2. In Section 3, we disclose the key role of astrocytes leading special neuron modes, called mixing mode, when we do not take electromagnetic induction into account. Distribution of different firing modes in parameter space is provided. In Section 4 , we compare both of astrocyte activities and electromag- netic induction and explore their different roles. Their dominated regions are illustrated in two-parameter space. Finally, conclusions are drawn in Section 5.

\section{Governing Equation: Neuron-Astrocyte Model under Electromagnet Field}

An improved ML model as a basic governing equation is selected to construct a model with consideration of electromagnetic induction and modulation of astrocytes. Membrane potential is determined not only by inward currents caused by $\mathrm{Ca}^{2+}$, delayed $\mathrm{K}^{+}$, and passive leak currents, respectively, but also by external stimulation, adjusted effect of astrocytes electromagnetic flux. The model is rewritten as follows [32],

$$
\begin{aligned}
C \frac{d v}{d t}= & -g_{\mathrm{Ca}} m_{\infty}\left(v-v_{\mathrm{Ca}}\right)-g_{K} w\left(v-v_{K}\right)-g_{L}\left(v-v_{L}\right)+I_{\text {slow }} \\
& +I_{\text {ext }}+I_{\text {ast }}+I_{\text {mag }}
\end{aligned}
$$

$\frac{d w}{d t}=\frac{\psi\left[w_{\infty}-w\right]}{\tau_{w}}$,

where

$$
\begin{aligned}
m_{\infty} & =0.5\left[1+\tanh \left(\frac{v-\bar{v}_{1}}{\bar{v}_{2}}\right)\right], \\
w_{\infty} & =0.5\left[1+\tanh \left(\frac{v-\bar{v}_{3}}{\bar{v}_{4}}\right)\right], \\
\tau_{w} & =\frac{1}{\cosh \left(v-\bar{v}_{3} / 2 \bar{v}_{4}\right)}, \\
\frac{d I_{\text {slow }}}{d t} & =\epsilon\left(v^{*}-v-\alpha I_{\text {slow }}\right) .
\end{aligned}
$$

$w$ is the second variable of the model which represents the fraction of open channel of $K^{+} \cdot g_{\mathrm{Ca}}, g_{K}$, and $g_{L}$ are constant ion channel conductances. $m_{\infty}, w_{\infty}$, and $\tau_{w}$ are functions governing the ion channel dynamics and partially determine the neuron firing dynamics. $v_{K},(K=1,2,3,4)$ are tuning parameters. $I_{\text {slow }}$ gives birth to bursting behavior, and the parameters $\epsilon$ and $\alpha$ greatly modulate the rhythm and transition of neuron bursting.

It is assumed that $\mathrm{IP}_{3}$ is decided by the neurotransmitter release $[T]$, and it controls $\mathrm{Ca}^{2+}$ concentration in cytoplasm. The change of calcium ion concentration consists of three parts: the $\mathrm{Ca}^{2+}$ flux from the endoplasmic reticulum (ER) to the cytosol $J_{\text {channel }}$, the pump flux from cytoplasm to ER $J_{\text {pump }}$, and the leakage flux from the ER to cytosol $J_{\text {leak }}$. Activities of astrocytes, characterized by fluctuation of $\mathrm{IP}_{3}$ and $\mathrm{Ca}^{2+}$ concentration, are described as follows:

$$
\frac{d\left[\mathrm{IP}_{3}\right]}{d t}=\frac{1}{\tau_{\mathrm{IP}_{3}}}\left(\left[\mathrm{IP}_{3}\right]^{*}-\left[\mathrm{IP}_{3}\right]\right)+r_{\mathrm{IP}_{3}}[T]
$$


TABLE 1: Temperature and wildlife count in the three areas covered by the study.

\begin{tabular}{lccccc}
\hline Parameter & Value & Unit & Parameter & Value & Unit \\
\hline$v_{1}$ & 6 & $\mathrm{~s}^{-1}$ & {$\left[\mathrm{IP}_{3}\right]^{*}$} & 0.16 & $\mu \mathrm{M}$ \\
$c_{1}$ & 0.185 & - & $r_{\mathrm{IP}_{3}}$ & 7.2 & $\mathrm{mMs}^{-1}$ \\
$g_{\mathrm{Ca}}$ & 1 & - & $v_{4}$ & 0.145 & - \\
$v_{\mathrm{Ca}}$ & 1 & - & $v_{2}$ & 0.11 & $\mathrm{~s}^{-1}$ \\
$v_{3}$ & 0.9 & $\mu \mathrm{Ms}^{-1}$ & $g_{K}$ & 2 & - \\
$v_{K}$ & -0.7 & - & $\theta_{s}$ & 0.2 & - \\
$g_{\mathrm{Ca}}$ & 1 & - & $d_{3}$ & 0.94 & $\mu \mathrm{M}$ \\
$d_{1}$ & 0.13 & $\mu \mathrm{M}$ & $d_{2}$ & 1.05 & $\mu \mathrm{M}$ \\
$g_{L}$ & 0.5 & - & $\sigma_{s}$ & 0.02 & - \\
$v_{L}$ & -0.5 & - & {$\left[\mathrm{Ca}^{2+}\right]_{\mathrm{th}}$} & 200 & $\mathrm{nM}$ \\
$c_{0}$ & -2 & $\mu \mathrm{M}$ & $d_{5}$ & 0.082 & $\mu \mathrm{M}$ \\
$a_{2}$ & 0.2 & $\mu \mathrm{Ms}^{-1}$ & $\psi$ & 1.15 & - \\
$\bar{v}_{1}$ & -0.01 & - & $\kappa$ & 0.5 & $\mathrm{~s}^{-1}$ \\
$\tau_{\mathrm{IP}}$ & 7 & $\mathrm{~s}$ & $\tau_{\mathrm{Ca}^{2+}}$ & 6 & $\mathrm{~s}$ \\
$\bar{v}_{2}$ & 0.15 & - & $\bar{v}_{3}$ & 0.1 & - \\
$\bar{v}_{4}$ & 0.145 & - & $k_{1}$ & 0.01 & - \\
$k_{2}$ & 0.5 & - & $\alpha$ & 0.1 & - \\
$\beta$ & 0.1 & - & & & \\
\hline & & & & &
\end{tabular}

$$
\begin{aligned}
{[T] } & =\frac{1}{1+\exp \left(-\left(v-\theta_{s}\right) / \sigma_{s}\right)}, \\
\frac{d\left[\mathrm{Ca}^{2}+\right]}{d t} & =-J_{\text {channel }}-J_{\text {pump }}-J_{\text {leak }}, \\
\frac{d q}{d t} & =\alpha_{q}(1-q)-\beta_{q} q,
\end{aligned}
$$

where

$$
\begin{aligned}
J_{\text {channel }} & =c_{1} v_{1} q_{\infty}^{3} n_{\infty}^{3} q^{3}\left(\left[\mathrm{Ca}^{2+}\right]-\left[\mathrm{Ca}^{2+}\right]_{\mathrm{ER}}\right), \\
J_{\text {pump }} & =\frac{v_{3}\left[\mathrm{Ca}^{2+}\right]^{2}}{k_{3}^{2}+\left[\mathrm{Ca}^{2+}\right]^{2}}, \\
J_{\text {leak }} & =c_{1} v_{2}\left(\left[\mathrm{Ca}^{2+}\right]-\left[\mathrm{Ca}^{2+}\right]_{\mathrm{ER}}\right),
\end{aligned}
$$

with

$$
\begin{aligned}
q_{\infty} & =\frac{\left[\mathrm{IP}_{3}\right]}{\left[\mathrm{IP}_{3}\right]+d_{1}}, n_{\infty}=\frac{\left[\mathrm{Ca}^{2+}\right]}{\left[\mathrm{Ca}^{2+}\right]+d_{5}}, \\
{\left[\mathrm{Ca}^{2+}\right]_{\mathrm{ER}} } & =\frac{c_{0}-\left[\mathrm{Ca}^{2+}\right]}{c_{1}}, \\
\alpha_{q} & =a_{2} d_{2} \frac{\left[\mathrm{IP}_{3}\right]+d_{1}}{\left[\mathrm{IP}_{3}\right]+d_{5}}, \beta_{q}=a_{2}\left[\mathrm{Ca}^{2+}\right] .
\end{aligned}
$$

Amiri et al. propose a phenomenological model to describe feedback to neurons from astrocytes in a form of function $f$ :

$$
\frac{d f}{d t}=\frac{-f}{\tau_{\mathrm{Ca}^{2+}}}+(1-f) \kappa \Phi\left(\left[\mathrm{Ca}^{2+}\right]-\left[\mathrm{Ca}^{2+}\right]_{\mathrm{th}}\right),
$$

where the Heaviside function $\Phi$ implies that gliotransmitter release is triggered by calcium inside the astrocytes only if its concentration is beyond a threshold $\left[\mathrm{Ca}^{2+}\right]_{\text {th }}$. The additional currents induced by astrocytes added on the neuron are then described by

$$
I_{\text {ast }}=\gamma f
$$

As to the effect of electromagnetic induction, the corresponding current introduced into the membrane potential is realized by memristor which bridges magnetic flux across the membrane and potential. $\rho(\phi)$ is the memory conductance of external current caused by magnetic flux, which is usually chosen as a nonlinear form. In summary, the model governing the magnetic induction is described as follows:

$$
\begin{gathered}
I_{\mathrm{mag}}=-k_{1} \rho(\phi) V, \\
\frac{d \phi}{d t}=V-k_{2} \phi, \\
\rho(\phi)=\alpha+3 \beta \phi^{2},
\end{gathered}
$$

where $k_{1}$ measures the feedback strength from electromagnetic induction and $k_{2}$ is natural decay rate in the assumed homogeneous medium. The values of parameters are listed in Table. 1.

\section{Mixing Neuron Firing Mode Selection Modulated by Astrocytes without Magnetic Flux}

The parameter $r_{\mathrm{IP}_{3}}$ reflects the activities of astrocytes corresponding to neuron firing by determining the production of $\mathrm{IP}_{3}$ in astrocyte cytoplasm triggered by neuron spikes. When membrane potential increases beyond a threshold $\theta_{s}$, the astrocytes begin to release $\mathrm{IP}_{3}$ to stimulate $\mathrm{Ca}^{2+}$ concentration enhancement. It has been proofed that $r_{\mathrm{IP}_{3}}$ production can reduce threshold of spontaneous oscillation of neuron firing, and when $r_{\mathrm{IP}_{3}}$ is large enough, the neuron could oscillate without any external stimulation [19]. It is reasonable that the results are considered as an evidence epilepsy can also be induced by abnormal astrocyte activities beside neuron firing disorder.

In order to highlight the role of astrocytes in our study, we first illustrate the dynamic role of $\mathrm{IP}_{3}$ production in modulating neuron firing in ML model without magnetic induction into account $\left(k_{1}=0\right)$. We provide the neuron spike with $\mathrm{Ca}^{2+}$ oscillation in a large span of parameter $r_{\mathrm{IP}_{3}}$ in Figure 1. The motion of neuron firing remains period-4 bursting until $r_{\mathrm{IP}_{3}}$ is greater than 40 , at which the system 

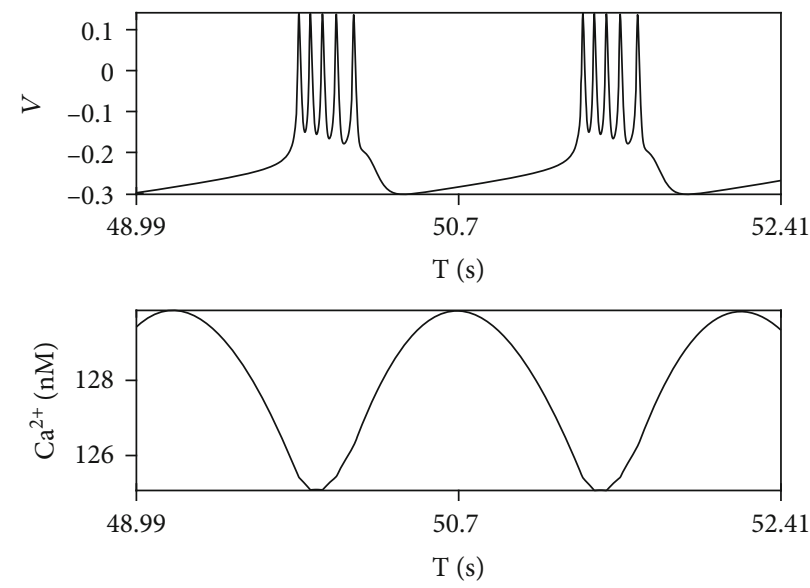

(a) $r_{\mathrm{IP}_{3}}=30 \mathrm{mMs}^{-1}$
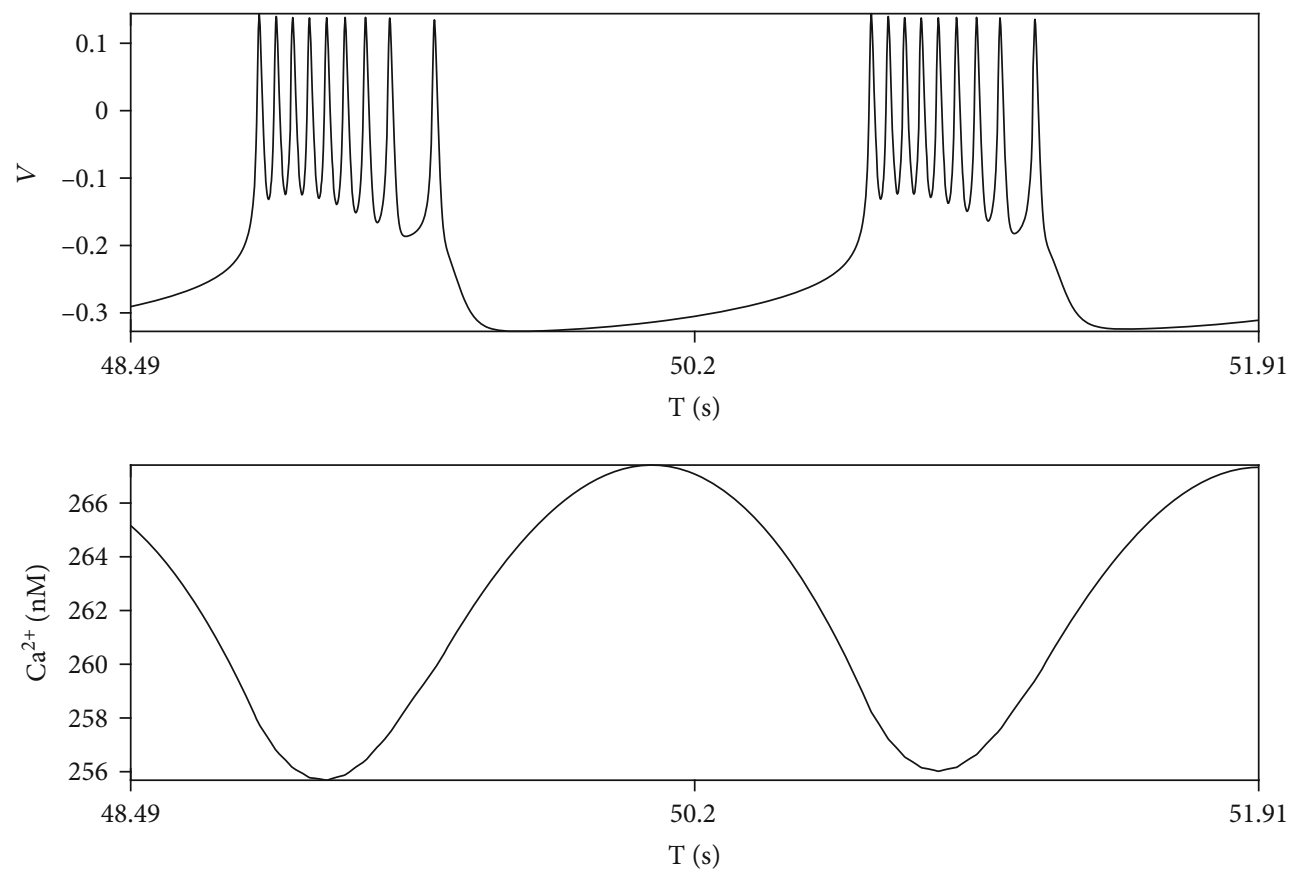

(b) $r_{\mathrm{IP}_{3}}=50 \mathrm{mMs}^{-1}$

Figure 1: Continued. 

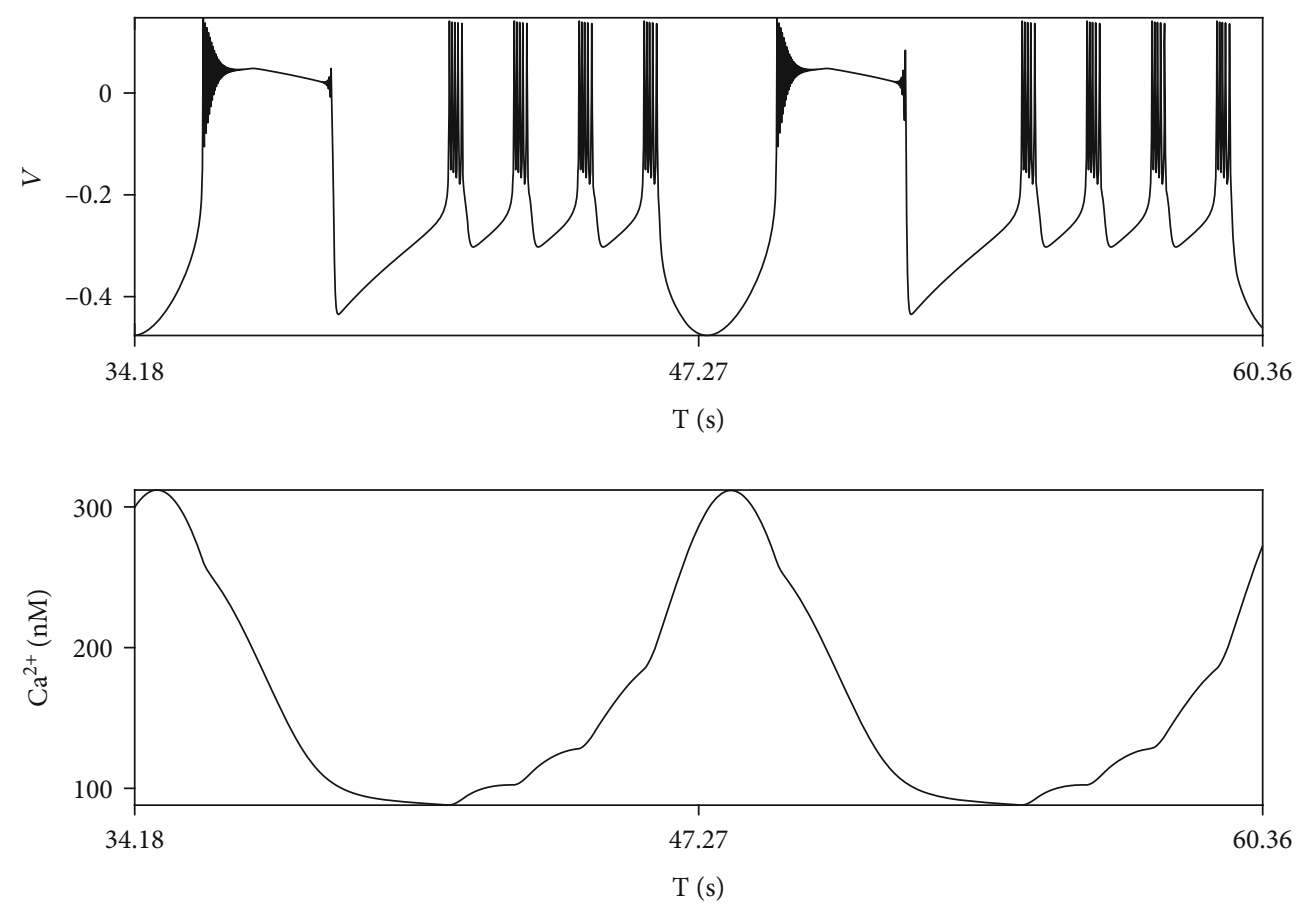

(c) $r_{\mathrm{IP}_{3}}=58 \mathrm{mMs}^{-1}$
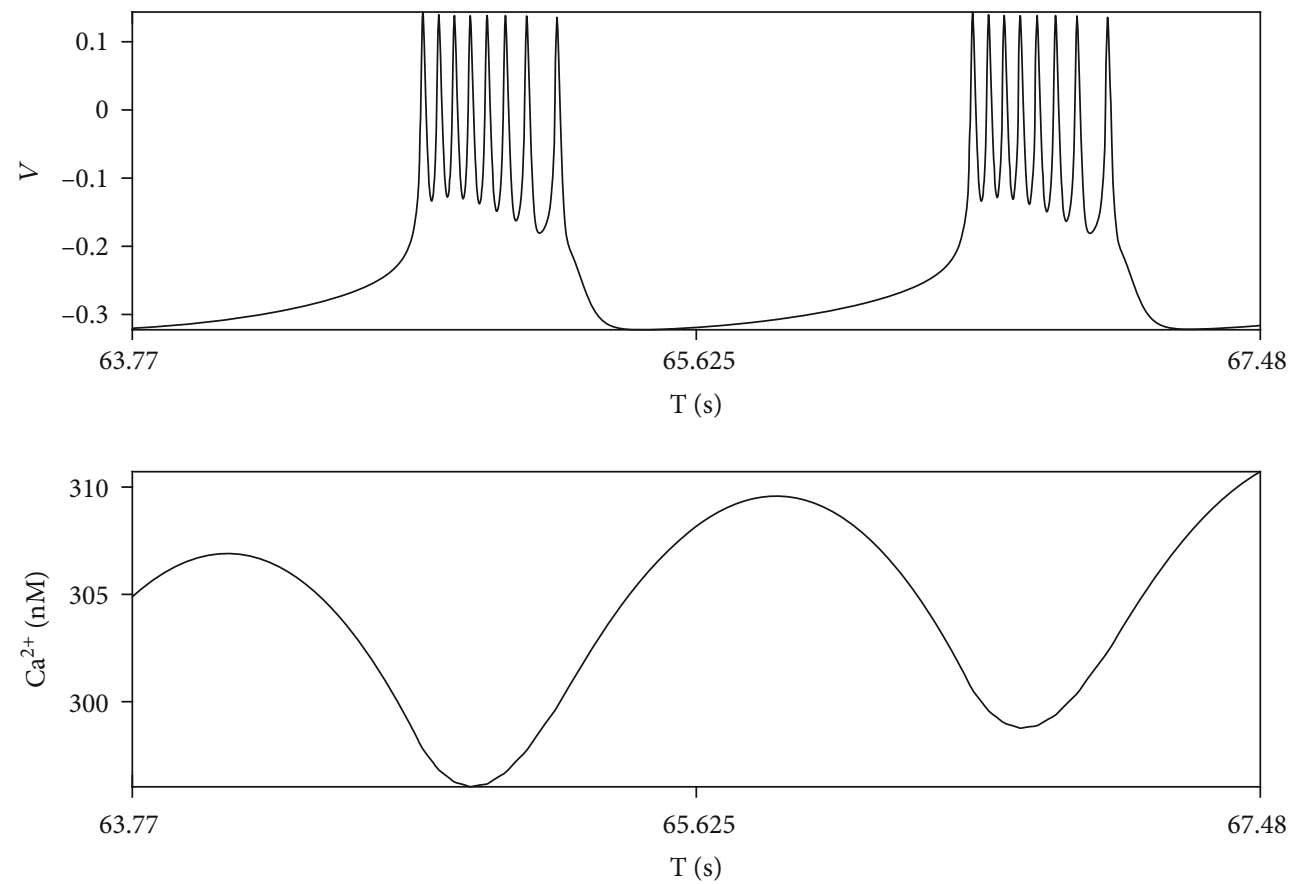

(d) $r_{\mathrm{IP}_{3}}=68 \mathrm{mMs}^{-1}$

FIgURe 1: Continued. 

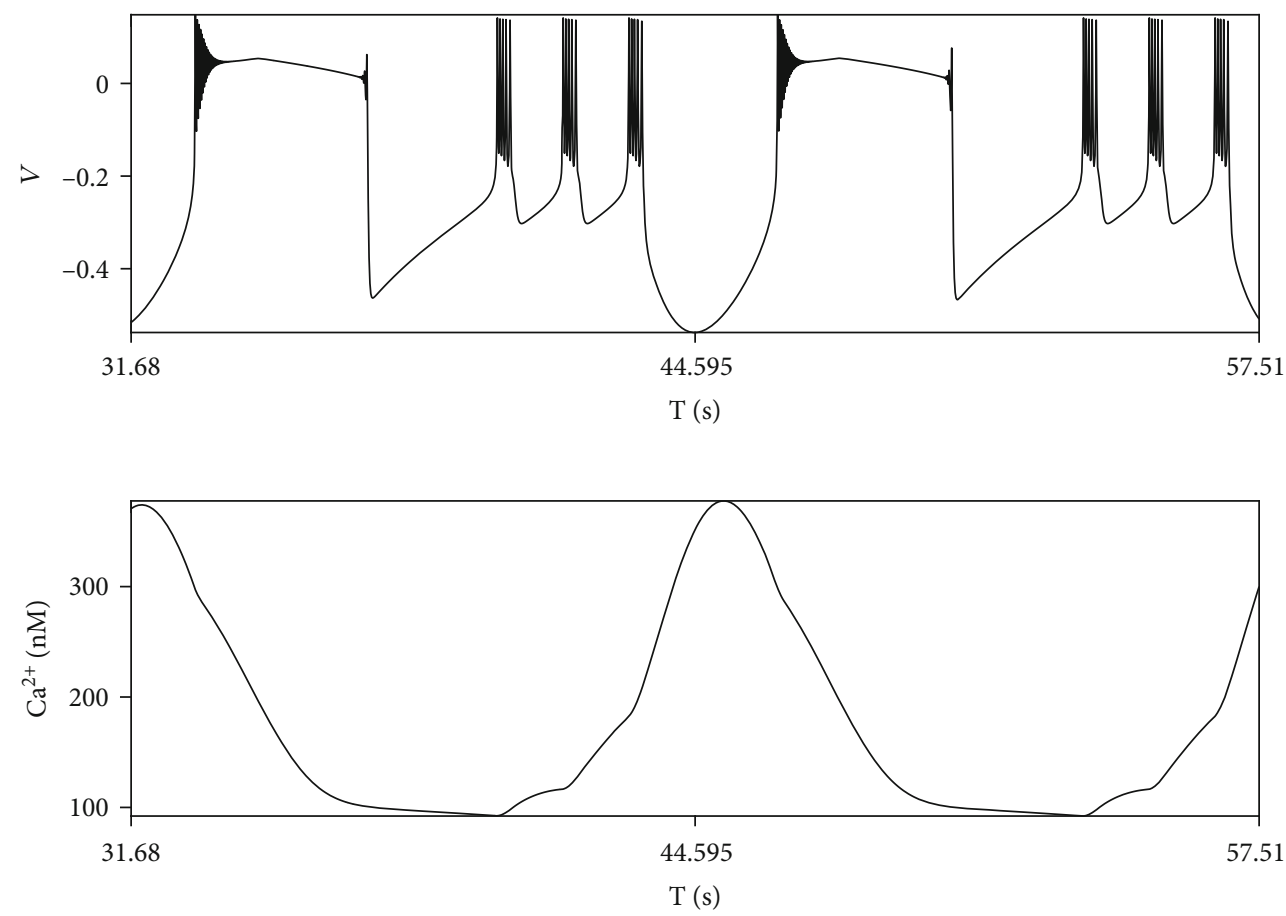

(e) $r_{\mathrm{IP}_{3}}=75 \mathrm{mMs}^{-1}$
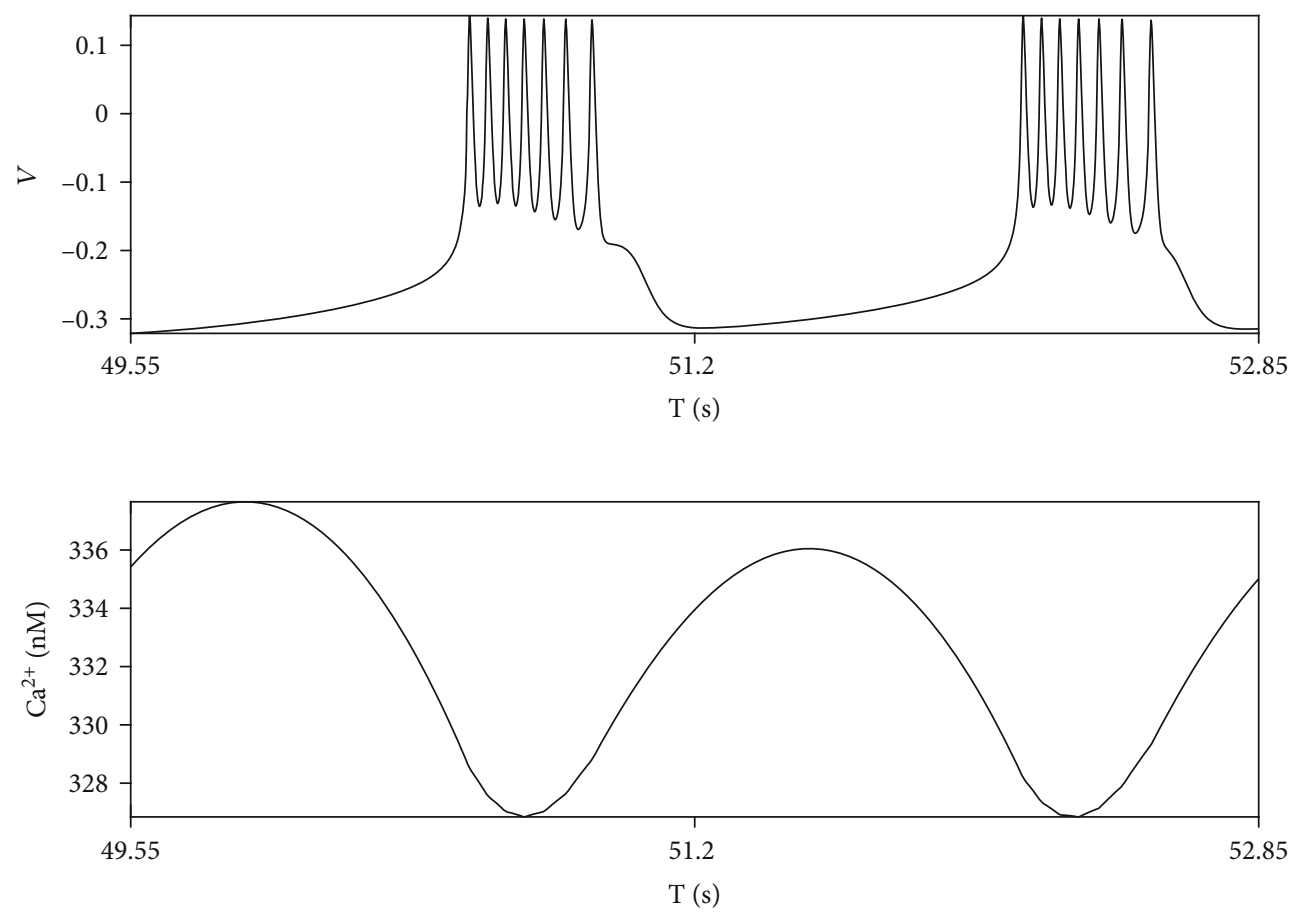

(f) $r_{\mathrm{IP}_{3}}=80 \mathrm{mMs}^{-1}$

Figure 1: Continued. 

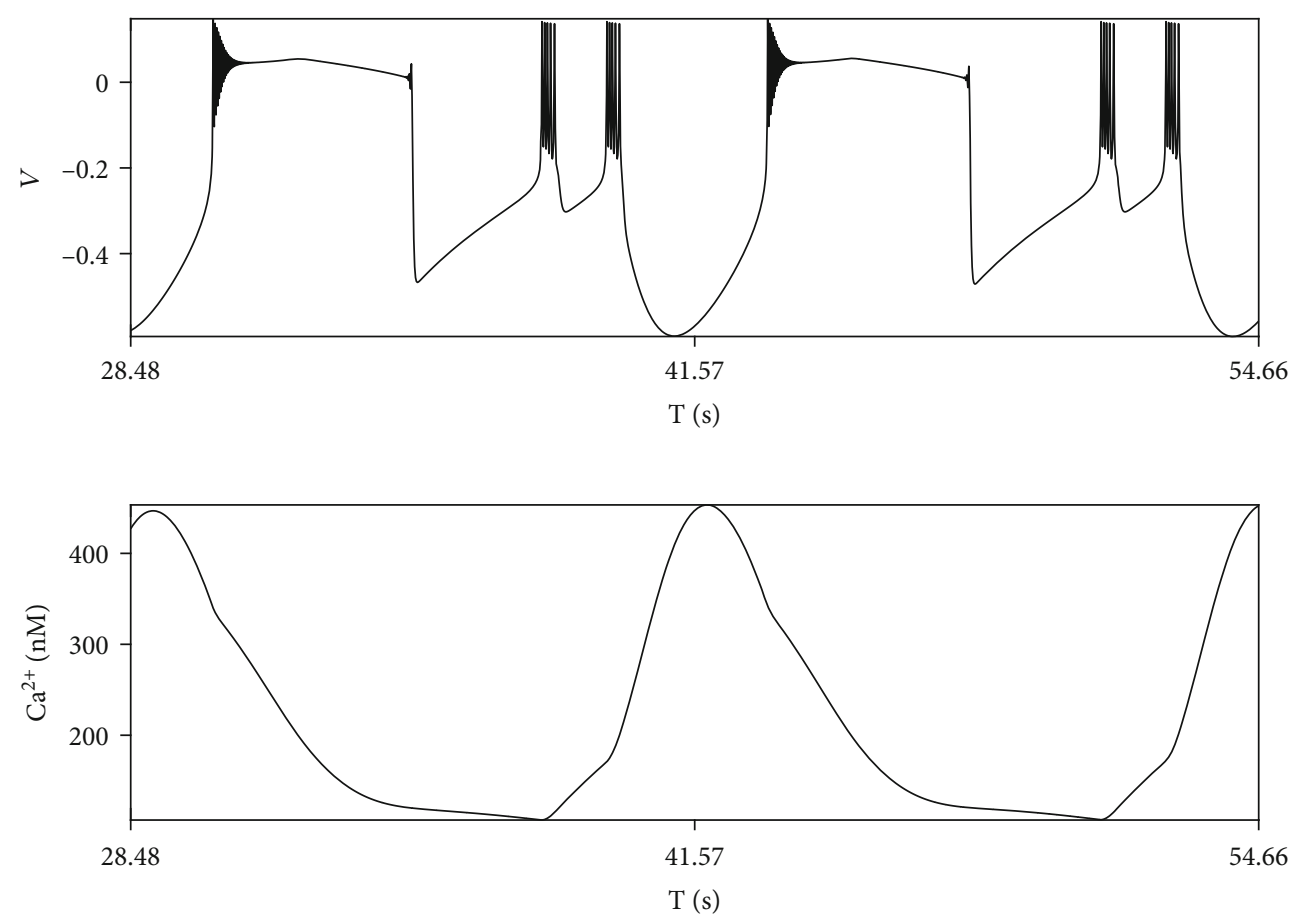

(g) $r_{\mathrm{IP}_{3}}=110 \mathrm{mMs}^{-1}$

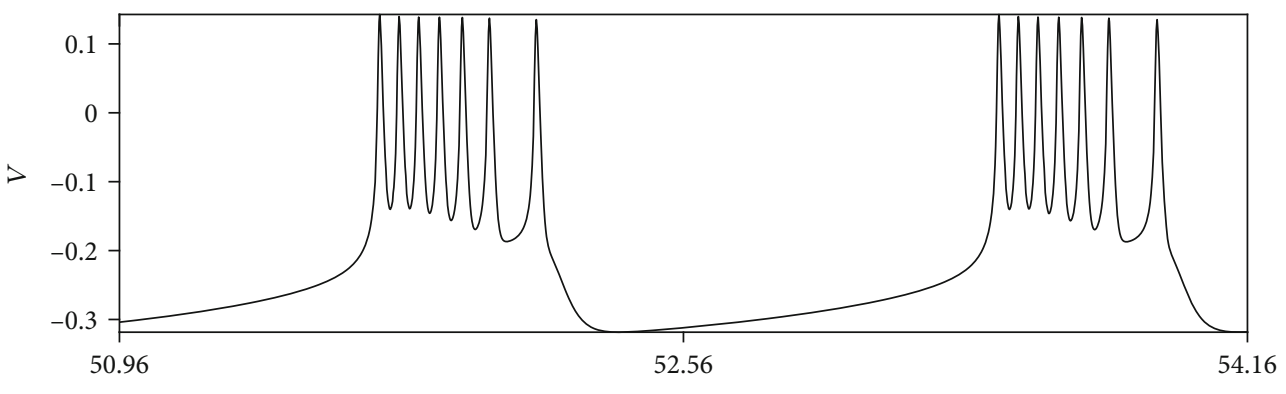

$\mathrm{T}(\mathrm{s})$

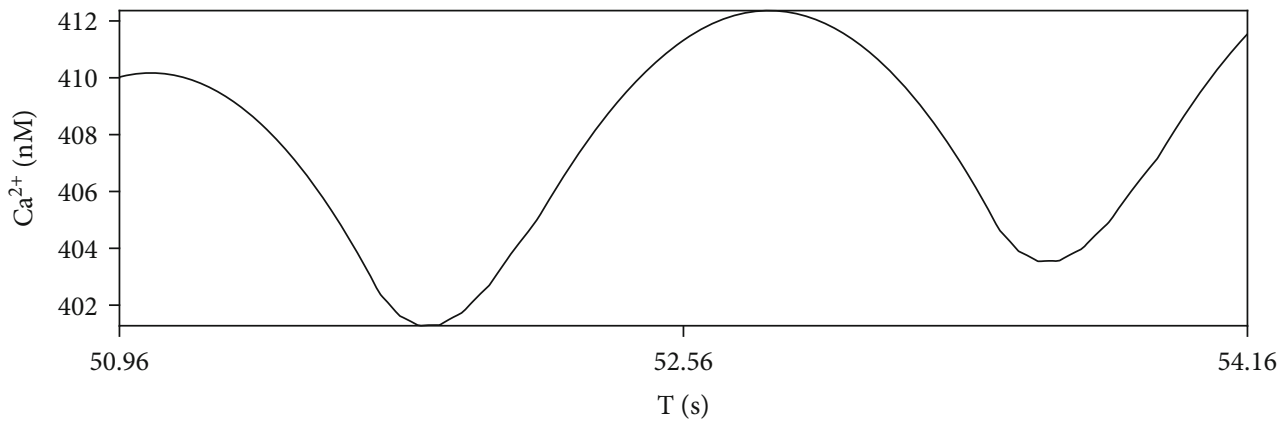

(h) $r_{\mathrm{IP}_{3}}=120 \mathrm{mMs}^{-1}$

FIGURE 1: The neuron firing modes and $\mathrm{Ca}^{2+}$ activities with no electromagnetic induction under different $r_{\mathrm{IP}}$.

transfers to period-9 bursting (Figures $1(\mathrm{a})$ and $1(\mathrm{~b})$ ). When $r_{\mathrm{IP}_{3}}$ continually increases a little, the motion of system enters a stage of alternation between periodic busting and depolarization blockage. In blockage, the membrane potential remains in a high level for a while and a spike cannot be sustained which is a typical abnormal firing mode in electro- graphic seizures [33]. Similarly, in the following interval of $r_{\mathrm{IP}_{3}}$, the motion switches back and forth between pure periodic bursting and the stage of alternation (between bursting and depolarization blockage). In Figures 1(c), 1(e), and $1(\mathrm{~g}), r_{\mathrm{IP}_{3}}$ locates in the intervals where motions of periodic bursting are interrupted by depolarization blockages. And 


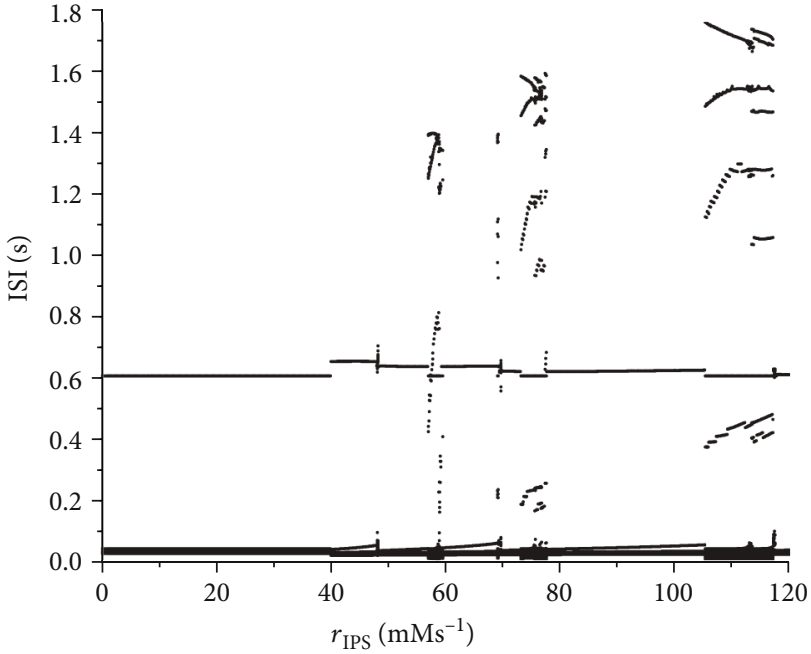

(a) ISI diagram

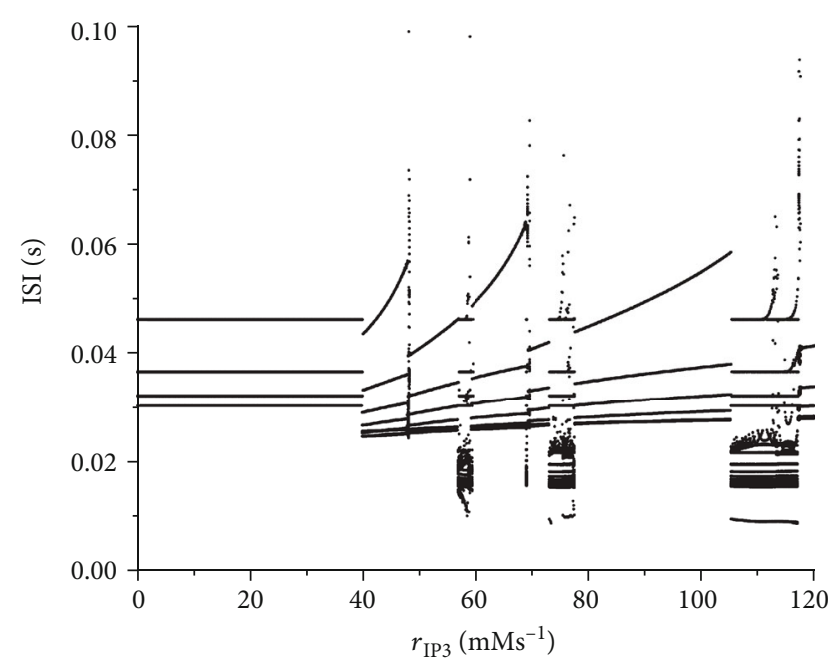

(b) Enlarged view of ISI diagram

FIGURE 2: The ISI diagram respect to $r_{\mathrm{IP}_{3}}$ and its enlarged view.

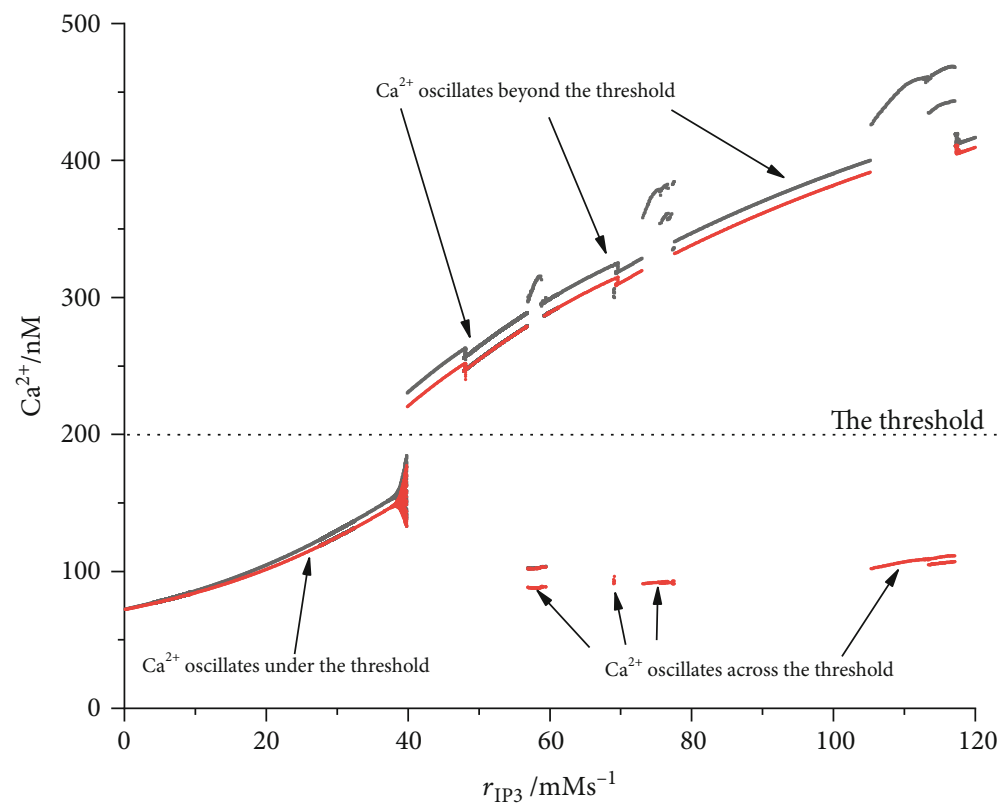

FIGURE 3: Crests and troughs of the oscillation of $\mathrm{Ca}^{2+}$ concentration respect to $r_{\mathrm{IP}_{3}}$. Black dots denote the crest, and red ones are troughs of the oscillation. The dashed line means the threshold.

the number of bursting between every two adjacent depolarization blockages decreases. The rest intervals represent period-4, period-9, period-8, period-6, and period-7 firing modes, respectively.

Interspike interval (ISI) diagram also confirms the transition from the alternation of two firing modes to depolarization blockage (see Figure 2). The intervals where the ISI burst out represent blockage and periodic bursting, because only in this mode can ISI reach every high values and occupy a very large span. The rest of them, denoted in the partial enlarged view, are firing modes of periodic bursting with different spike numbers.
Compared to the evolution of $\mathrm{Ca}^{2+}$, we confirm that every time when concentration penetrates downward the threshold, the end of depolarization blockage is discovered. When $\mathrm{Ca}^{2+}$ concentration is beneath the threshold, the current denoting the astrocyte activities turns off. On contrary, if the minimum of $\mathrm{Ca}^{2+}$ concentration is beyond the threshold, the current always turns on. Both of the two cases correspond to periodic bursting. However, as threshold locates inside the span of $\mathrm{Ca}^{2+}$ oscillation, the current alternatively and continuously turns on and off. Only in this case is the mixing firing mode observed in which depolarization blockage and periodic bursting appears alternatively. We plot the 

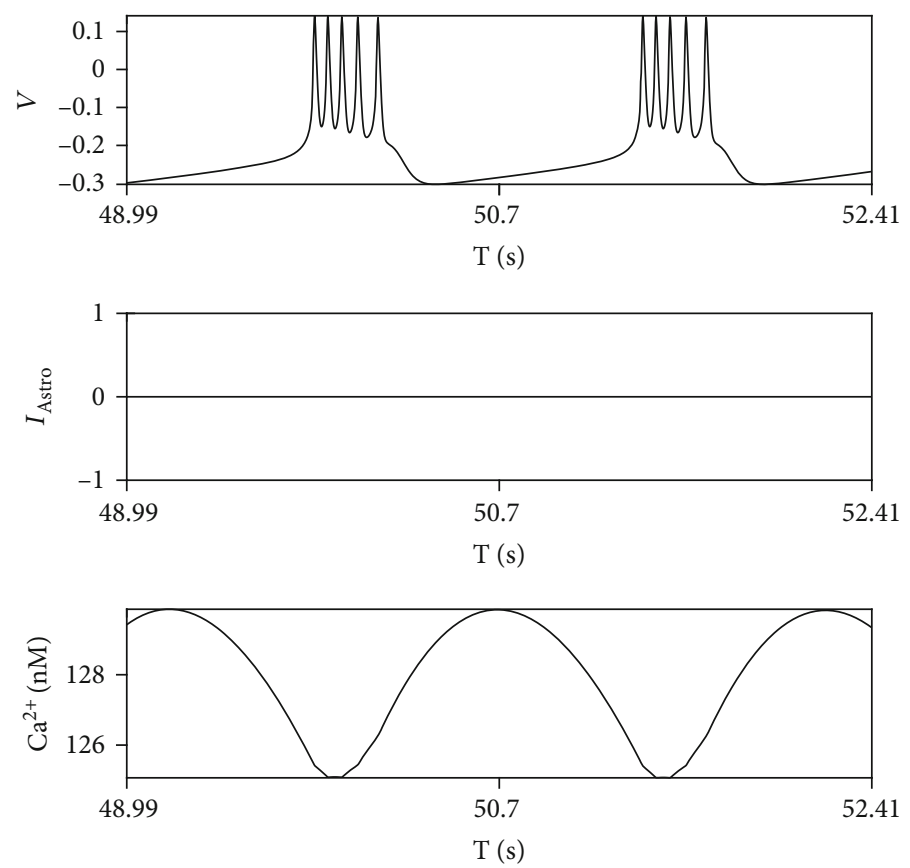

(a) $r_{\mathrm{IP}_{3}}=30 \mathrm{mMs}^{-1}$

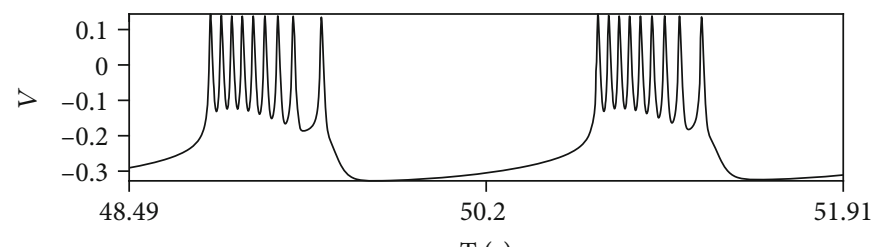

$\mathrm{T}(\mathrm{s})$
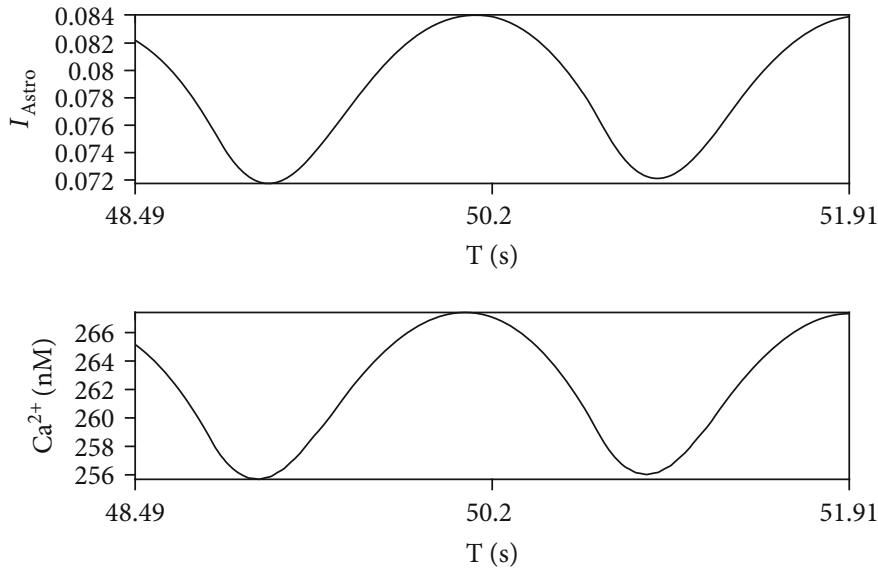

(b) $r_{\mathrm{IP}_{3}}=50 \mathrm{mMs}^{-1}$

Figure 4: Continued. 

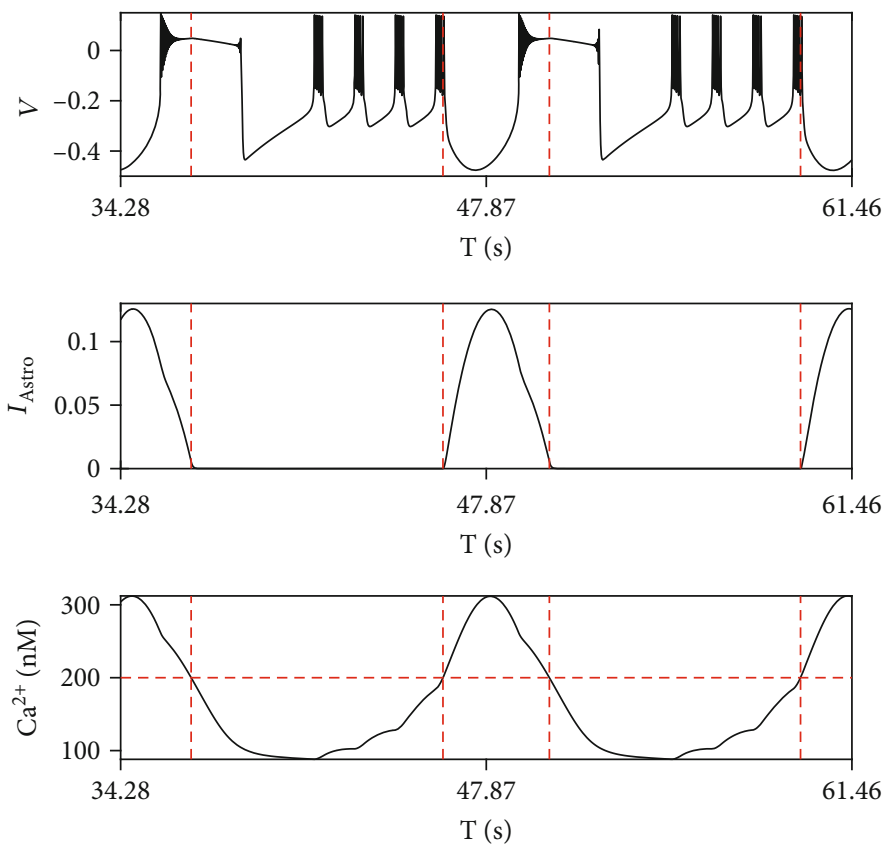

(c) $r_{\mathrm{IP}_{3}}=58 \mathrm{mMs}^{-1}$

Figure 4: Membrane potential compared with feedback current and $\mathrm{Ca}^{2+}$ activities in (a) $\mathrm{Ca}^{2+}$ is under threshold $\left(r_{\mathrm{IP}_{3}}=30 \mathrm{mMs}^{-1}\right),(\mathrm{b}) \mathrm{Ca}^{2+}$ oscillates across threshold $\left(r_{\mathrm{IP}_{3}}=50 \mathrm{mMs}^{-1}\right)$, and (c) $\mathrm{Ca}^{2+}$ oscillates above the threshold $\left(r_{\mathrm{IP}_{3}}=58 \mathrm{mMs}^{-1}\right)$.

crests and troughs of the oscillation of $\mathrm{Ca}^{2+}$ concentration compared to the threshold to represent the three cases in Figure 3.

The current applied to the membrane potential from astrocytes $I_{\text {ast }}$ definitely plays an important role in mode selection of neuron firing. In order to further specify the magnificence of astrocytes, membrane potential compared with astrocyte feedback current $I_{\text {ast }}$ in three type of cases at different $r_{\mathrm{IP}_{3}}$, (i) $\mathrm{Ca}^{2+}$ is under the threshold $\left(r_{\mathrm{IP}_{3}}=\right.$ $\left.30 \mathrm{mMs}^{-1}\right)$, (ii) $\mathrm{Ca}^{2+}$ oscillates across the threshold $\left(r_{\mathrm{IP}_{3}}=\right.$ $50 \mathrm{mMs}^{-1}$ ), and (iii) $\mathrm{Ca}^{2+}$ oscillates above the threshold $\left(r_{\mathrm{IP}_{3}}=58 \mathrm{mMs}^{-1}\right) . I_{\text {ast }}$ keeps zero, and $\mathrm{Ca}^{2+}$ oscillates under the threshold (Figure 4(a)), which means that the astrocyte do not release gliotransmitters and is absent in modulating neuron firing. When $\mathrm{Ca}^{2+}$ oscillates beyond the threshold, $I_{\text {ast }}$ begins to oscillate with a very small fluctuation of amplitude, and neuron remains in bursting state (Figure 4(b)). And it seems that the number of spikes in every periodic bursting in this case is more than that when $\mathrm{Ca}^{2+}$ is under the threshold. In the mixing mode $\mathrm{Ca}^{2+}$ oscillates in a large span of amplitude as $r_{\mathrm{IP}_{3}}=58 \mathrm{mMs}^{-1}$ (Figure 4(c)). $I_{\text {ast }}$ shrinks to zero accompanied with that $\mathrm{Ca}^{2+}$ drops to the threshold. At the same time, the oscillation of membrane potential attenuates quickly and remains in a high level until it falls to a negative value. During the interval in which $I_{\text {ast }}$ keeps zero $\left(\mathrm{Ca}^{2+}\right.$ is under the threshold), periodic bursting generates and is interrupted by that $\mathrm{Ca}^{2+}$ increases and passes the threshold to trigger the release of gliotransmitters.

\section{Neuron Firing Modulated Both with Astrocytes and under Electromagnetic Flux}

Before elucidating the role of electromagnetic induction, we specify the effect of $I_{\text {slow }}$ in generating and controlling neuron firing. Periodic bursting vanishes, and neuron keeps in resting state when there is no $I_{\text {slow }}(\epsilon=0$, Figure 5(a)). Periodic bursting or oscillation exists when $\epsilon$ is a small positive value. Span of interspike interval between every cluster decreases rapidly accompanied with the fact that spike number in every period also decreases, when we increase $\epsilon$. Periodic bursting becomes periodic oscillation as $\epsilon$ passes a critical value. The scenario is illustrated by membrane potential at different values of $\epsilon$ (Figures 5(b)-5(d)). The upper branch in ISI diagram (Figure 5(e)) denotes the intervals between clusters, and lower one denotes the intervals between spikes inside the clusters. The two branches converge to one branch at the critical value of $\epsilon$ where periodic oscillation replaces the periodic bursting. ISI between bursting, denoted by upper branch, shrinks in the power law, which means that span of ISI and the parameter $\epsilon$ satisfy ISI $=A * e^{-\lambda}$. The truth is illustrated by a line in double logarithmic coordinate with $\lambda=0.8639$. In this study, $\epsilon$ is kept in 0.002 in order to guarantee the existence of periodic bursting.

Beside feedback current from astrocytes, electromagnetic induction also plays a key role in modulating neuron firing. In the model we improved, $k_{1}$ is the most important parameter to measure the magnitude of feedback current. We select three different cases to elucidate the role of electromagnetic induction: (a) only induction adjusts neuron firing $r_{\mathrm{IP}_{3}}=0$ 


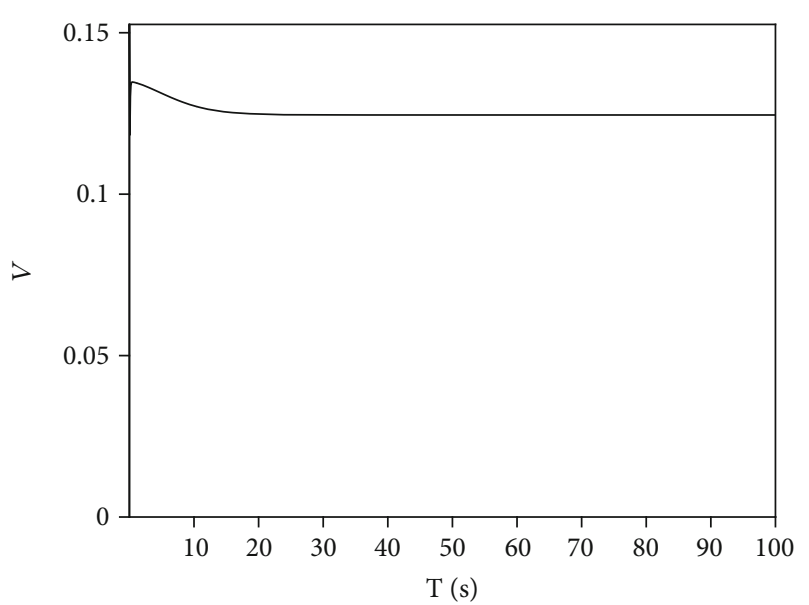

(a) Membrane potential as $\epsilon=0$

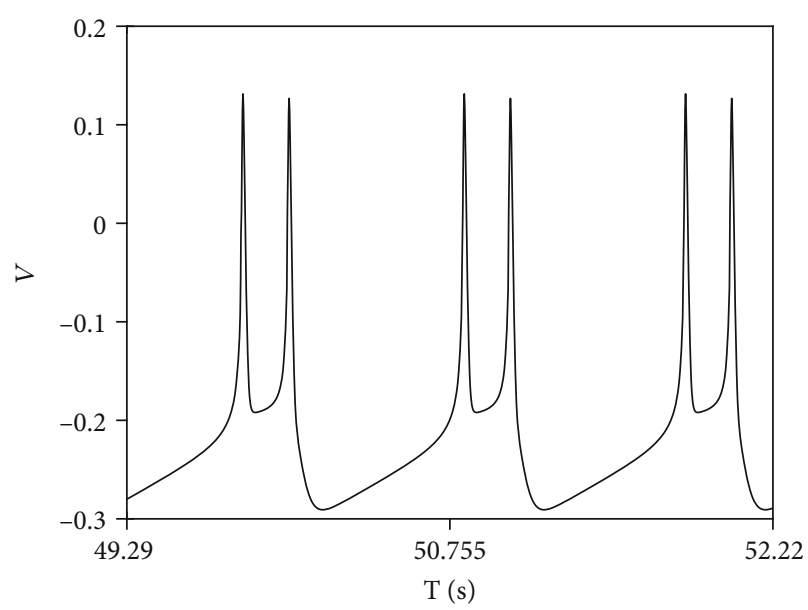

(c) Membrane potential as $\epsilon=0.005$

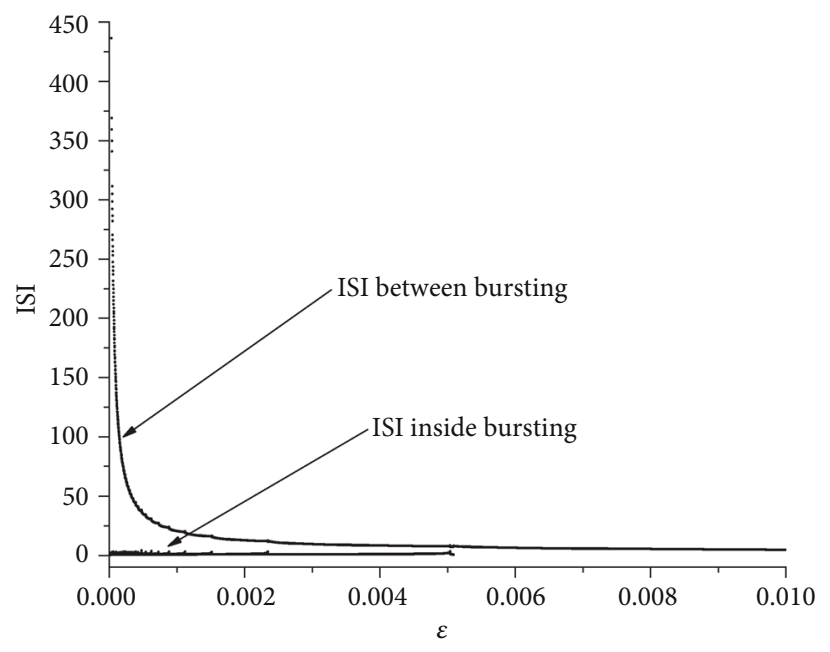

(e) ISI as varying $\epsilon$

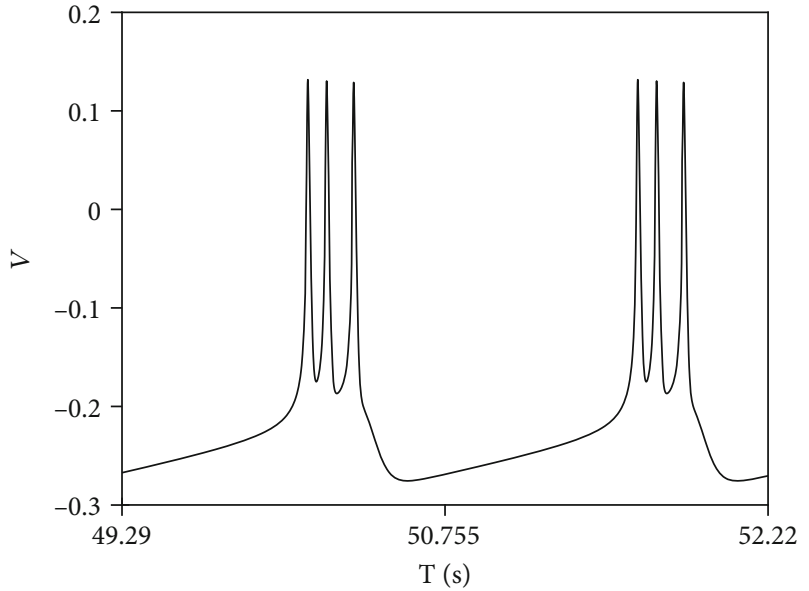

(b) Membrane potential as $\epsilon=0.002$

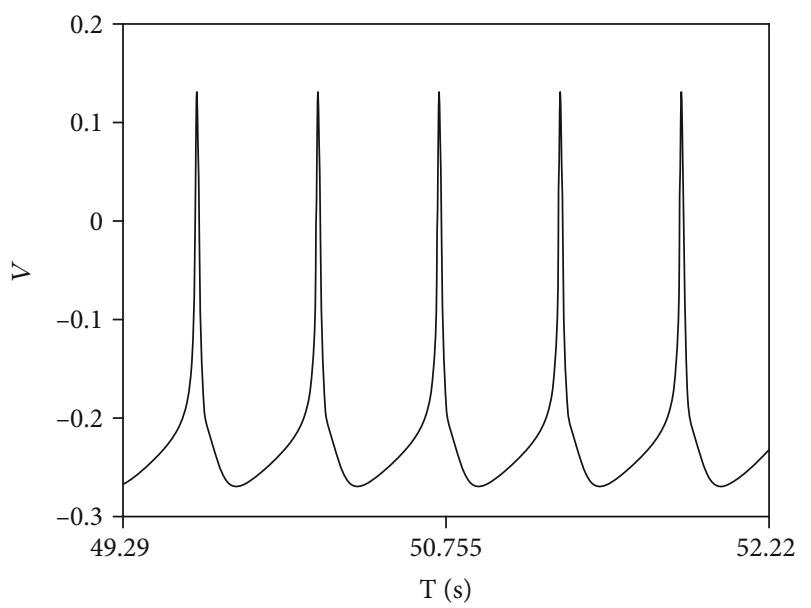

(d) Membrane potential as $\epsilon=0.008$

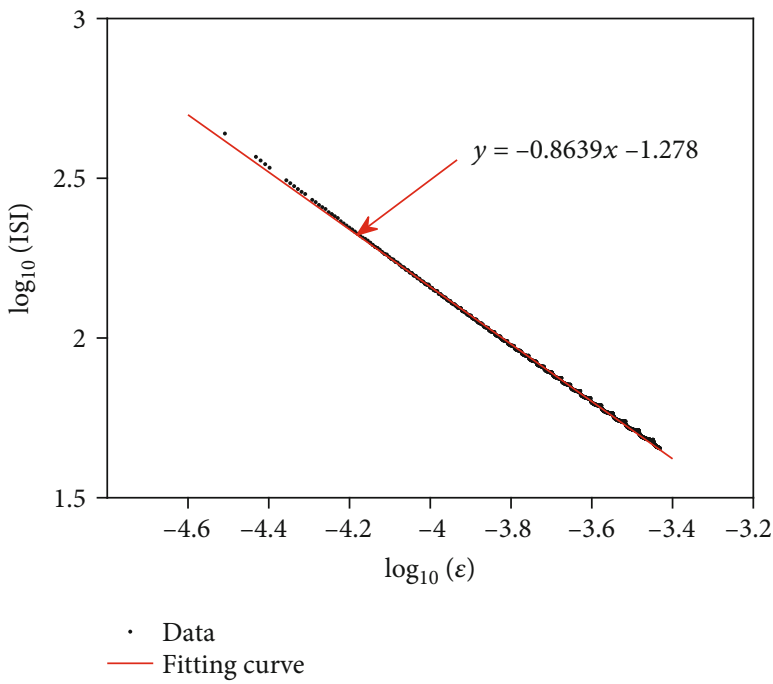

(f) Interval between bursting in double logarithmic coordinate

FIgURE 5: Membrane potential and ISI diagram varying $\epsilon$ with electromagnetic induction $\left(k_{1}=0.5\right)$ considered but without astrocytes $\left(r_{\mathrm{IP}_{3}}=0 \mathrm{mMs}^{-1}\right)$. 

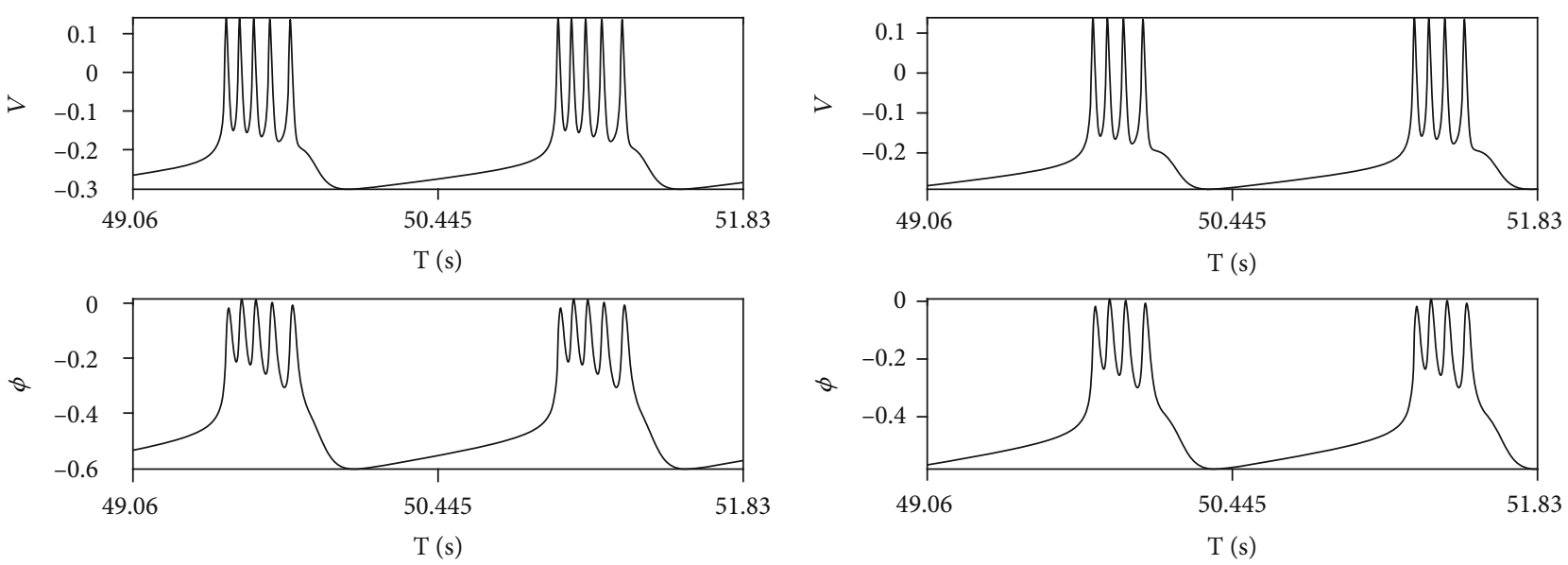

(a) $k_{1}=0$

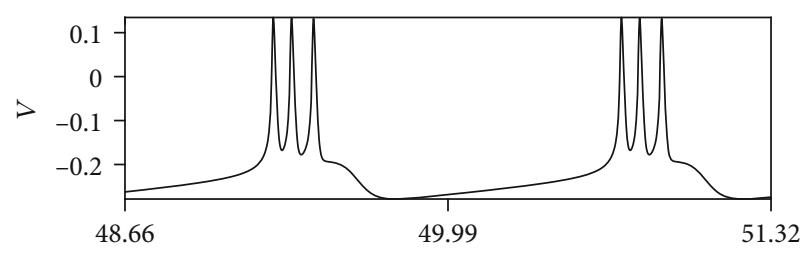

(b) $k_{1}=0.15$
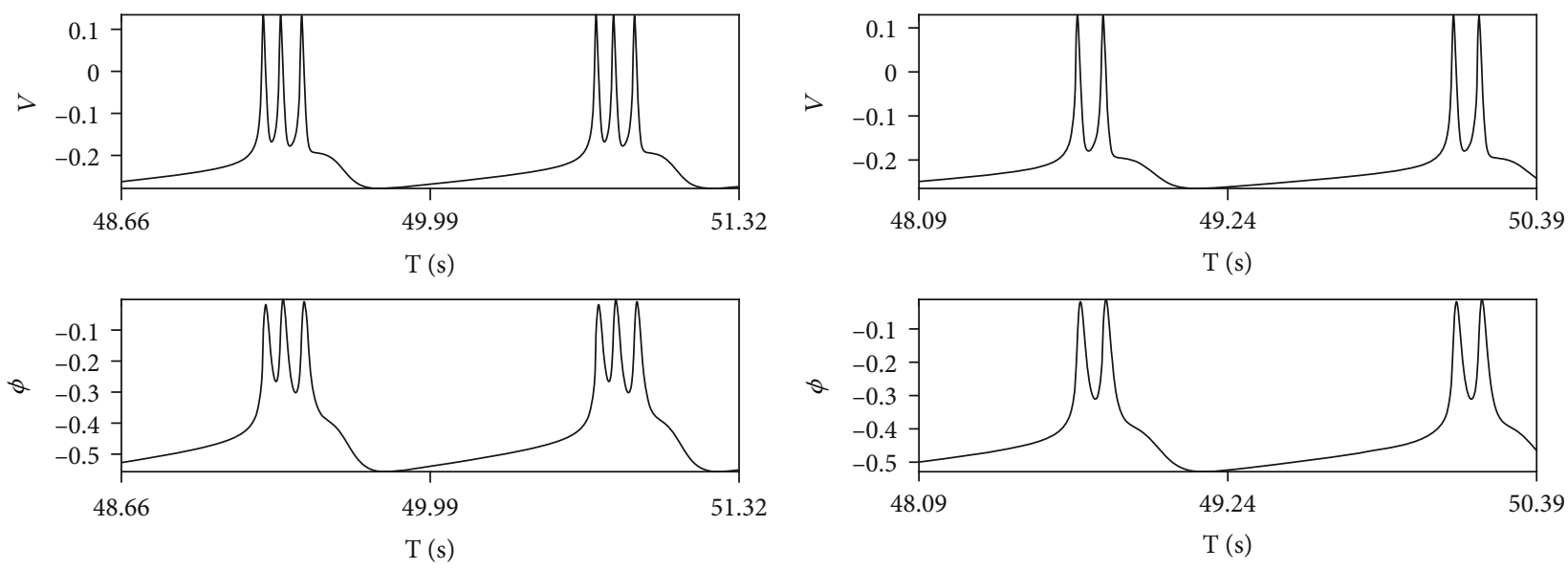

(c) $k_{1}=0.35$

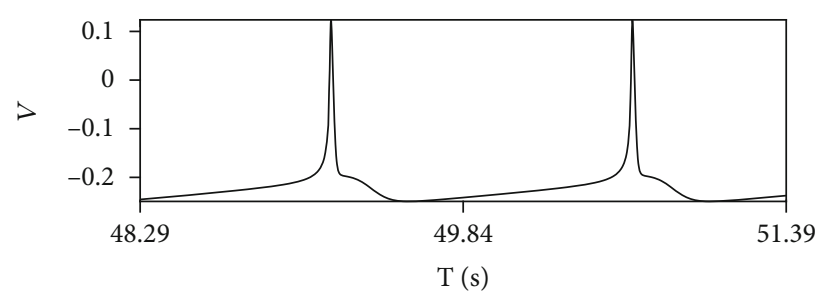

(d) $k_{1}=0.60$
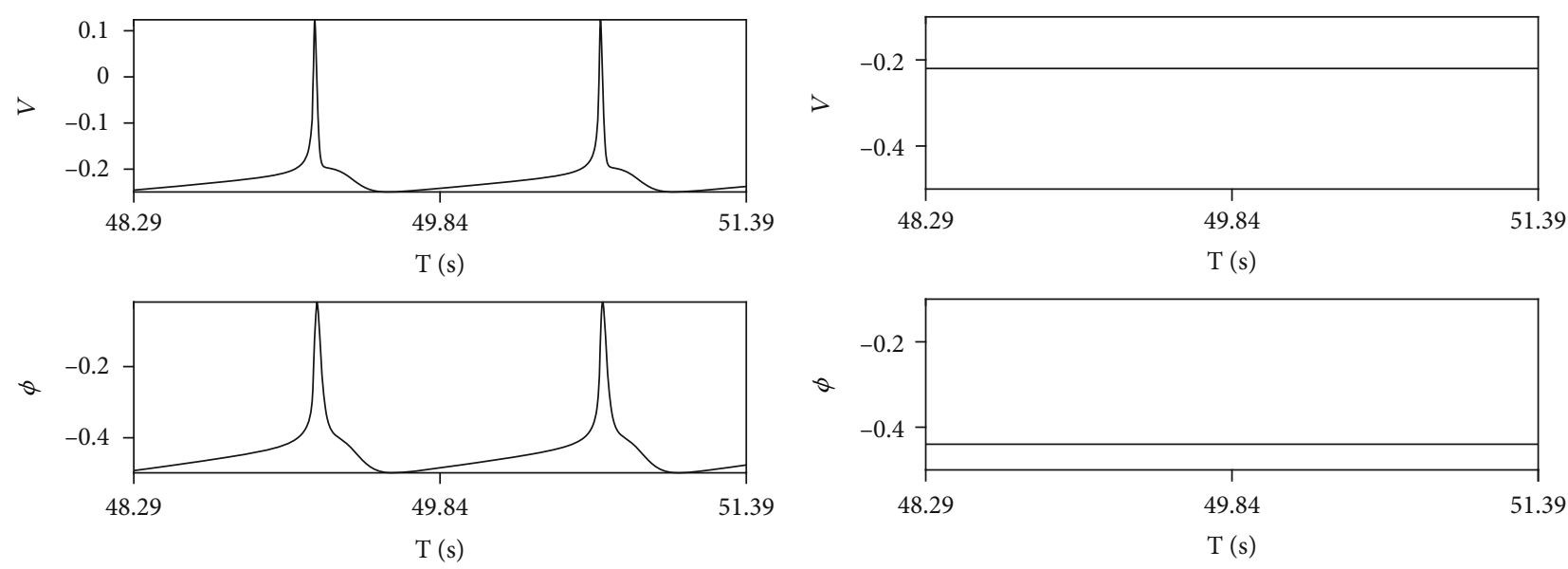

(e) $k_{1}=0.90$

(f) $k_{1}=1.10$

Figure 6: The neuron firing modes and magnetic flux $\phi$ without astrocyte activities.

$\mathrm{mMs}^{-1}$, (b) we start from periodic clustering as $r_{\mathrm{IP}_{3}}=80 \mathrm{mMs}^{-1}$ and vary $k_{1}$, and (c) $r_{\mathrm{IP}_{3}}=100 \mathrm{mMs}^{-1}$ is chosen at which alternation of periodic clustering and depolarization blockage occurs at $k_{1}=0$.

Without oscillation of $\mathrm{Ca}^{2+}$ concentration, depolarization blockage cannot exist in firing mode, leading that only periodic bursting is left as varying amplitude of feedback current $k_{1}$. Transition from periodic bursting to resting state is achieved by increasing $k_{1}$ with setting $r_{\mathrm{IP}_{3}}=0 \mathrm{mMs}^{-1}$ (see Figure 6). Number of spikes during one period decreases leading that period-5, period-4, period-3, period-2, period1 bursting, and resting state are displayed in turn. 

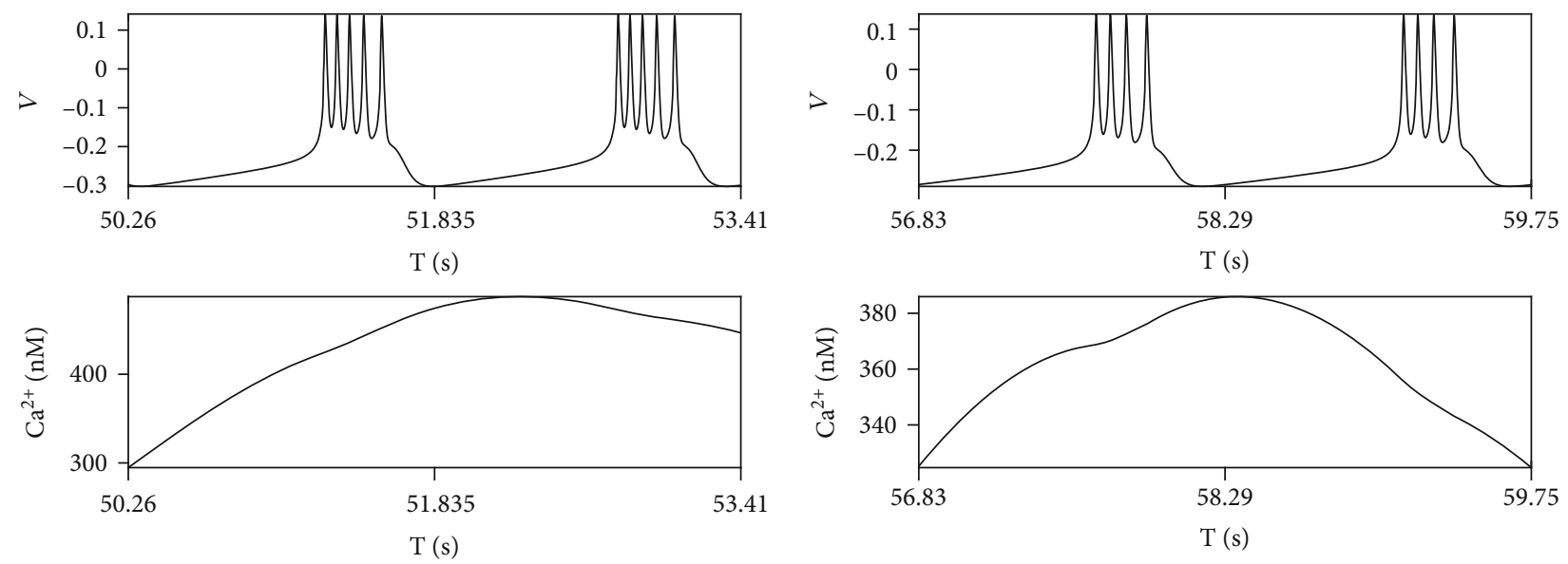

(a) $k_{1}=0$

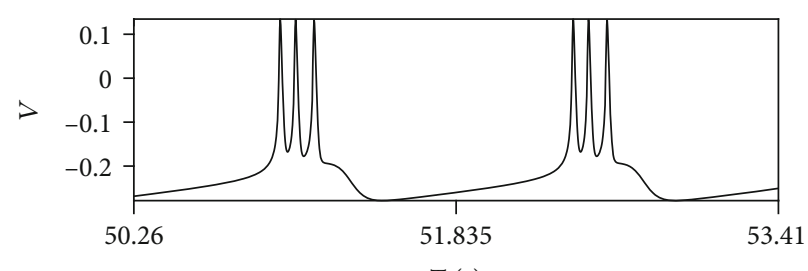

(b) $k_{1}=0.20$
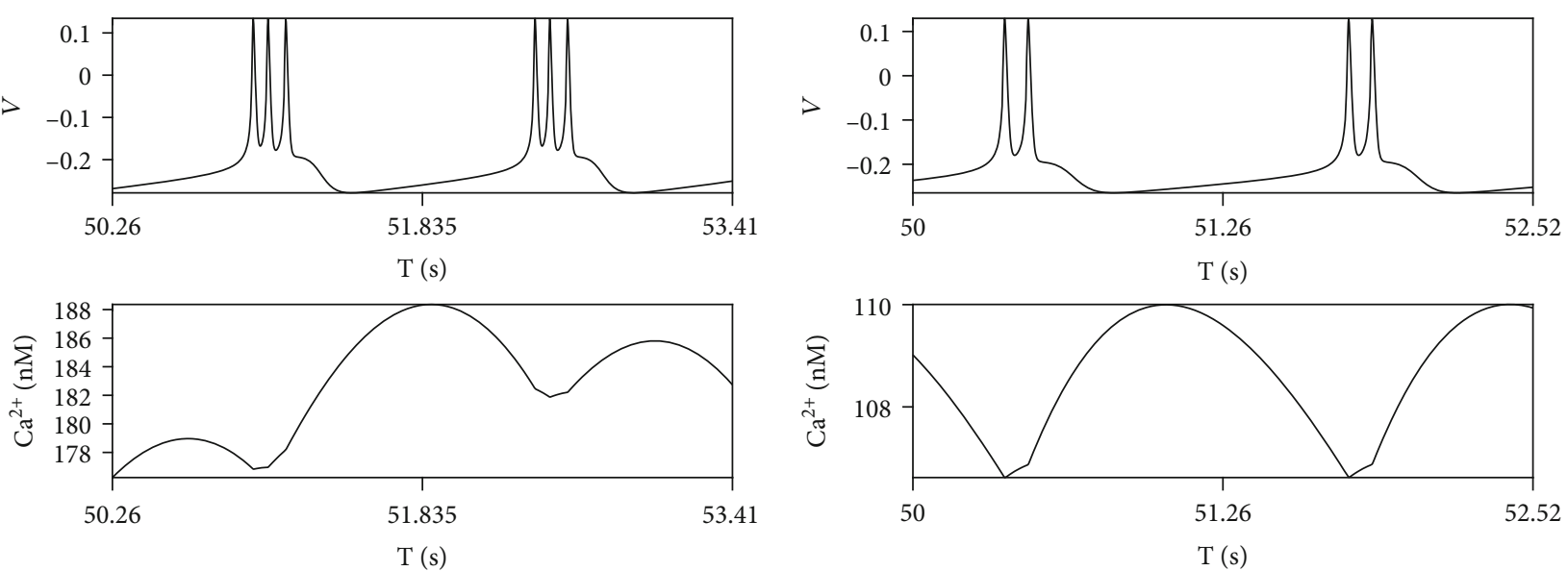

(c) $k_{1}=0.35$

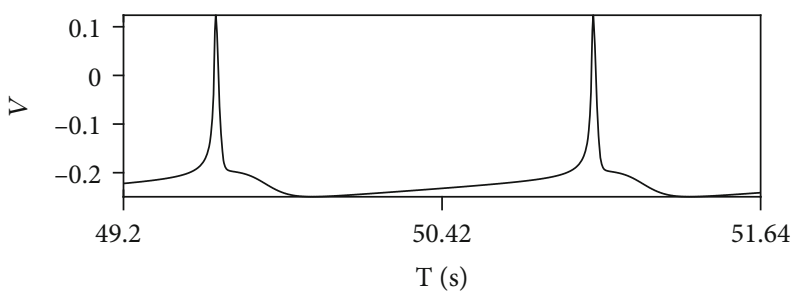

(d) $k_{1}=0.60$
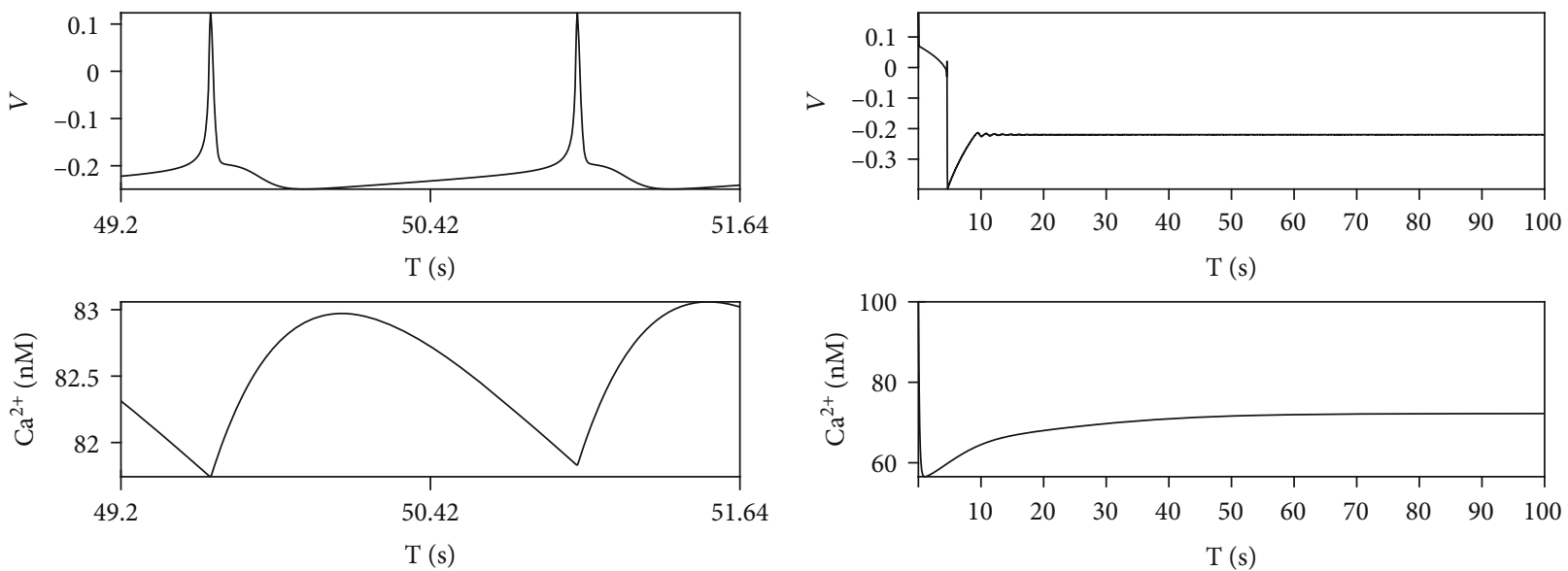

(e) $k_{1}=0.90$

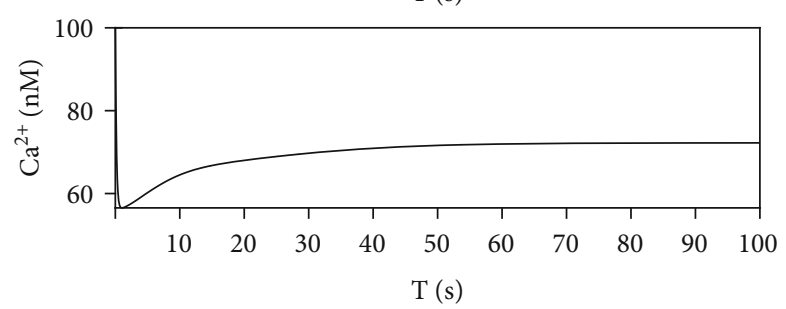

(f) $k_{1}=1.10$

FIGURE 7: The neuron firing modes and $\mathrm{Ca}^{2+}$ activities as $r_{\mathrm{IP}_{3}}=80 \mathrm{mMs}^{-1}$.

There is no mixing mode when $r_{\mathrm{IP}_{3}}=80 \mathrm{mMs}^{-1}$, see Figure 7. $\mathrm{Ca}^{2+}$ concentration oscillates either beyond the threshold (Figures 7(a) and 7(b)) or beneath the threshold (Figures $7(\mathrm{c})-7(\mathrm{e})) . \mathrm{Ca}^{2+}$ concentration does not oscillate around the threshold which guarantee that depolarization blockage cannot be appear in firing modes. Resting firing dominates the firing mode as $k_{1}$ is greater than 1 (Figure $7(\mathrm{f})$ ).

With $r_{\mathrm{IP}_{3}}=100 \mathrm{mMs}^{-1}$, different neuron firing modes represent in Figure 8 as varying $k_{1}$. Mixing mode is observed when $k_{1}=0$, but it suddenly vanishes when $k_{1}$ is little bit more than 0 . Mixing modes cannot be discovered only except 

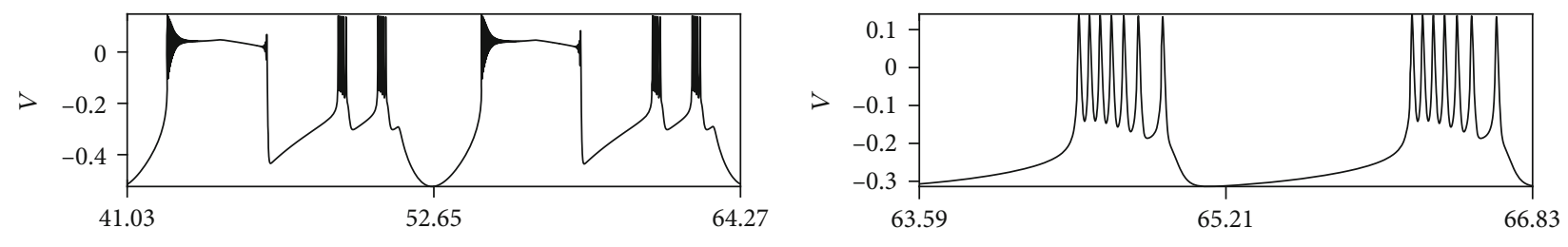

$\mathrm{T}(\mathrm{s})$
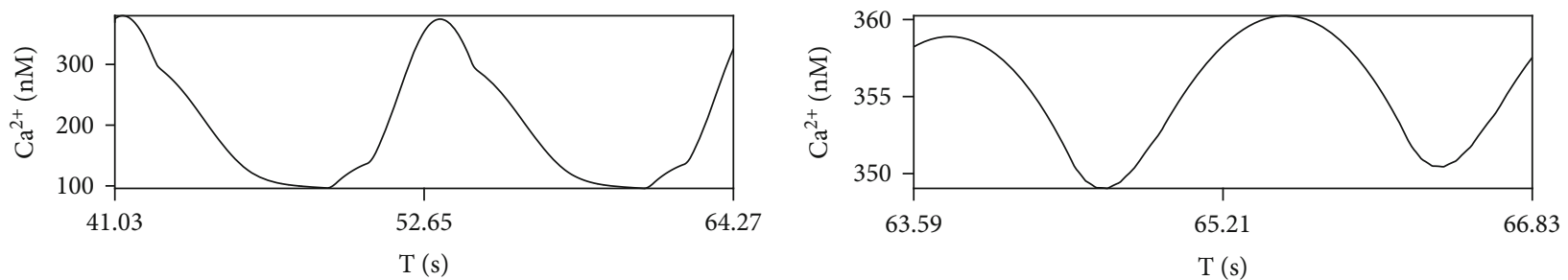

(a) $k_{1}=0$

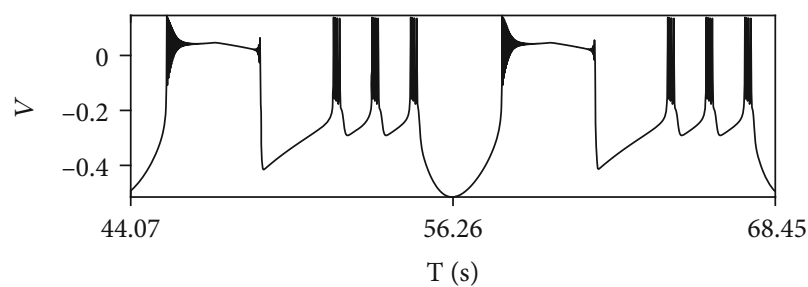

(b) $k_{1}=0.10$
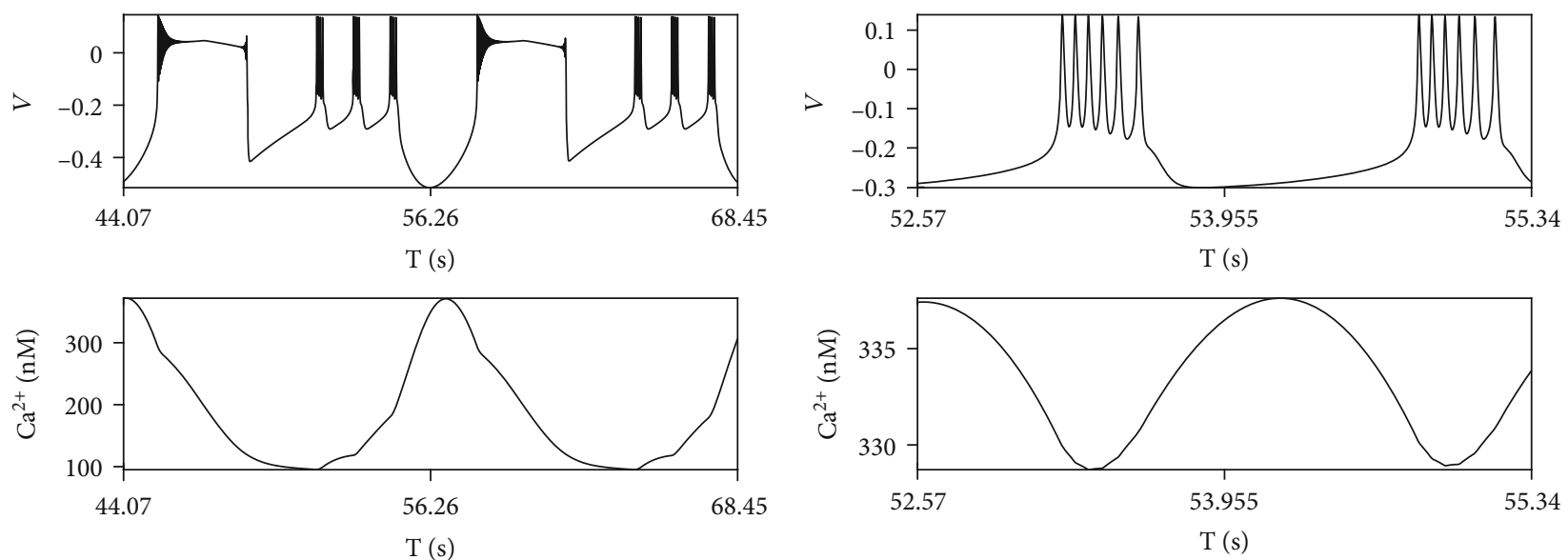

(c) $k_{1}=0.15$

(d) $k_{1}=0.20$
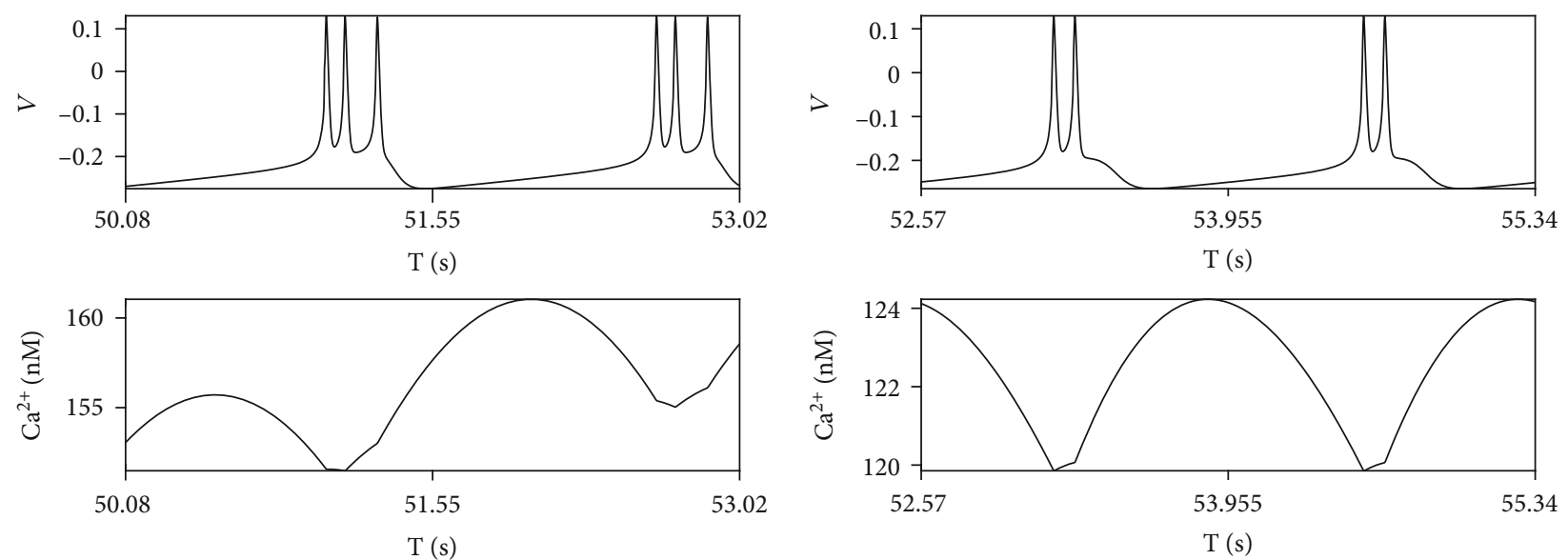

(e) $k_{1}=0.55$

(f) $k_{1}=0.60$

Figure 8: Continued. 

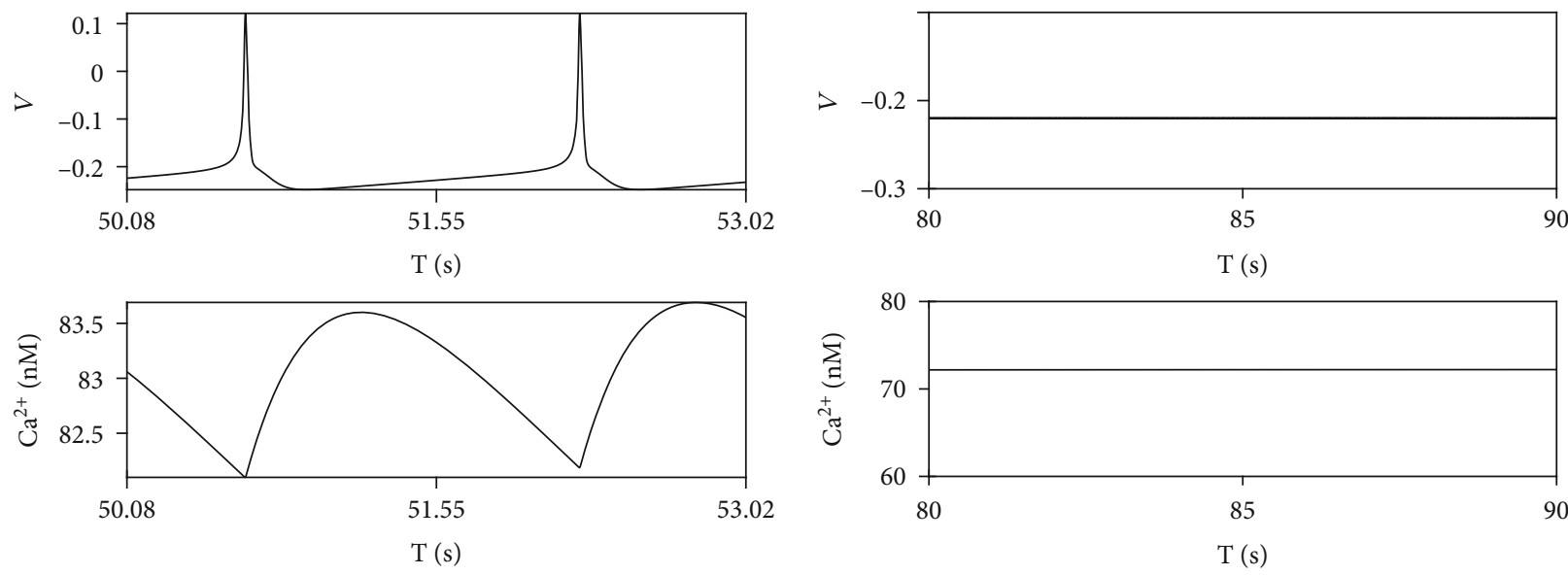

(g) $k_{1}=1.00$

(h) $k_{1}=1.2$

FIgURE 8: The neuron firing modes and $\mathrm{Ca}^{2+}$ activities as $r_{\mathrm{IP}_{3}}=100 \mathrm{mMs}^{-1}$.

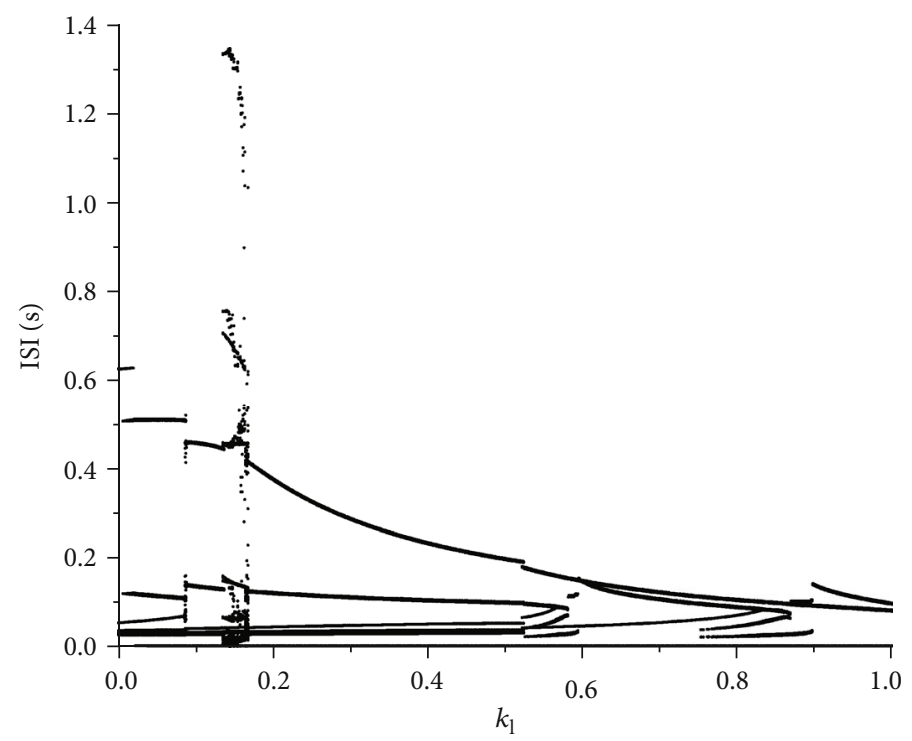

FIGURE 9: The ISI diagram respect to $k_{1}$ with $r_{\mathrm{IP}_{3}}=100 \mathrm{mMs}^{-1}$.

a small interval $0.14<k_{1}<0.17$, leading periodic bursting in firing mode. Number of spikes in one period decreases with respect to $k_{1}$. Period-7 bursting becomes period-6 as $k_{1}$ becomes 0.20 from 0.10 ; then, it reduces to period- 3 , period-2, and at last period-1 when $k_{1}$ gets approach to 1.0. When $k_{1}$ is greater than 1 , spike neuron firing disappears, and only oscillation with very small amplitude under threshold exists.

ISI diagram also illustrates the same statement by reducing span of ISI values with increasing $k_{1}$ (see Figure 9). Obviously, the current from electromagnetic induction introduced into membrane potential play a key role of suppressing spike neuron firing. Mixing mode is rarely observed in neuron firing, and periodic bursting vanishes, and firing mode becomes resting state when amplitude of current is large enough.
We also provide the two-parameter space to show distribution of mixing mode, which illustrate the great ability that electromagnetic induction suppresses the depolarization blockage or mixing mode. As illustrated in Figure 10, mixing mode only exists in some isolate parameter regions when $k_{1}<0.22$. The rest of regions are dominated by periodic bursting and resting state.

In order to further specify the inhibition role of electromagnetic induction, we provide the bifurcation diagram with $k_{1}$ as the control parameter (Figure 11). The limit circle (denoted by blue lines) becomes a stable focus (denoted by black line) via saddle-node bifurcation. This transition means that violent oscillations dominated by a limit circle when $k_{1}$ is small enough, degenerate into small oscillations or resting states when the motion is in the basin of stable focus as increasing $k_{1}$. 


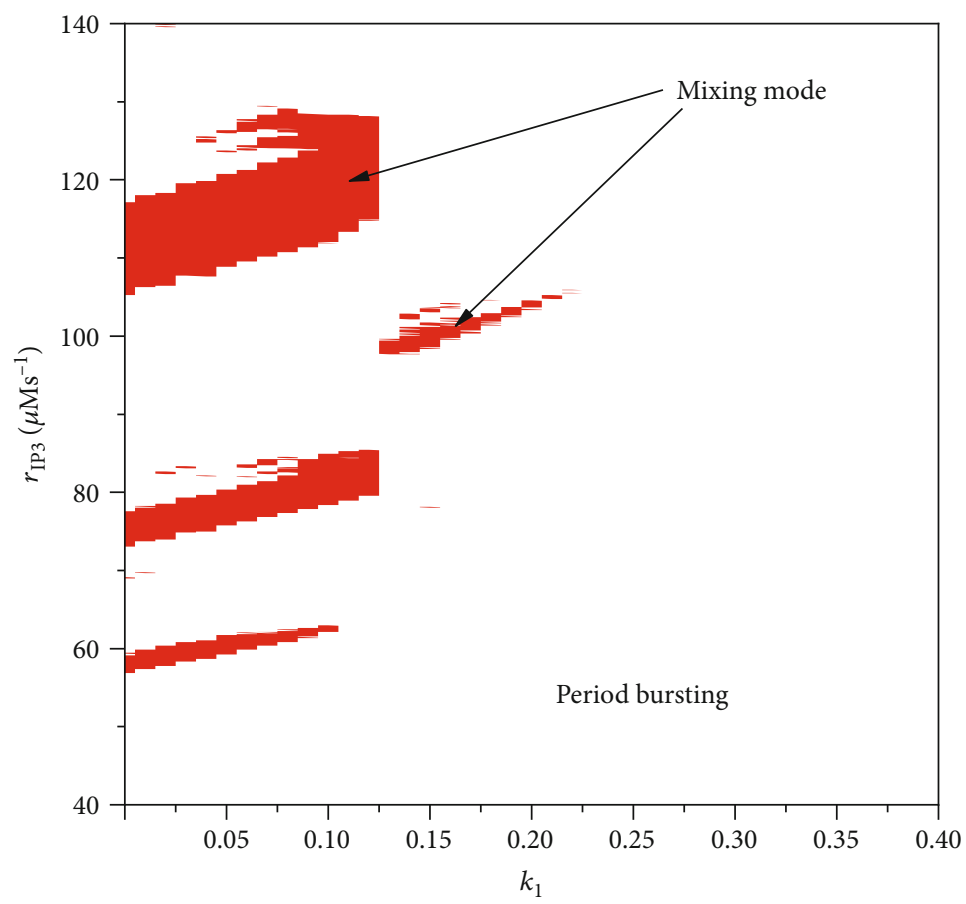

FiguRE 10: Regions dominated by mixing mode or periodic bursting in parameter space. Red regions denote the mixing mode, and white region is periodic bursting.

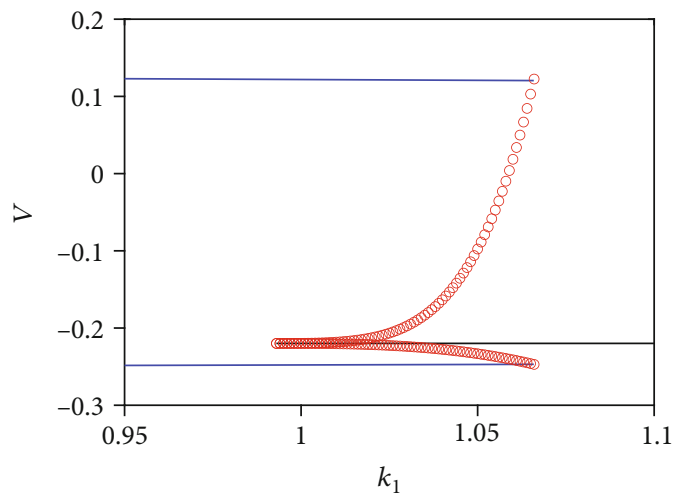

FIGURE 11: Bifurcation diagram as varying amplitude of feedback current from electromagnetic induction $k_{1}$.

\section{Conclusions and Prospects}

In this paper, we numerically explore scenario of neuron firing mode selection among mixing mode, periodic bursting, and resting state. Mixing mode is an alternation stage between periodic bursting and depolarization blockage. Depolarization blockage appears only when $\mathrm{Ca}^{2+}$ concentration goes up through the threshold and ends when $\mathrm{Ca}^{2+}$ concentration penetrates down through the threshold. Therefore, mixing mode dominates the firing mode only when concentration of $\mathrm{Ca}^{2+}$ oscillate across value of threshold.

When there is no electromagnetic induction adjusting neuron firing mode, introduction of astrocytes could induce depolarization blockage into neuron firing, with combination of periodic bursting to compose mixing mode. Mixing mode and periodic bursting distribute alternatively in the parameter space. With respect to $r_{\mathrm{IP}_{3}}$, the interval between every two adjacent depolarization blockages shrinks, and the number of periodic clustering firing decreases. But when the amplitude of feedback current is large enough, mixing mode cannot be observed in neuron firing because $\mathrm{Ca}^{2+}$ concentration is beyond the threshold.

The role of slow current is also studied. The number of spikes in one period decreases, and span of ISI between clusters shrinks in a power law when increasing the amplitude of slow current $\epsilon$ and finally brings periodic bursting into periodic oscillation. Electromagnetic induction plays an important role of suppressing neuron firing. As amplitude of feedback current $k_{1}$ increasing, mixing mode shrinks gradually, and $k_{1}$ is greater than 1 , the mixing mode is completely vanishes. Distribution in two-parameter space also indicates the same statement. The inhibition role of electromagnetic field is explained by saddle-node bifurcation which brings violent oscillations into small ones or resting state.

Our research focuses on neuron firing modes transition and dynamical properties of them in the improved ML model, with consideration of both astrocytes and electromagnetic induction. We compare different role of them and study numerically their effects of modulation on neuron firing. Therefore, the results are of great potential significance on control strategies of mode selection by controlling amplitude of feedback currents introduced by both of the two factors and even on therapy of refractory diseases including suppressing epilepsy and realizing defibrillation. 


\section{Data Availability}

The data used to support the findings of this study are available from the corresponding author upon request.

\section{Conflicts of Interest}

The authors declare no competing financial interests.

\section{Acknowledgments}

The project is supported by the Special Fund for Basic Scientific Research of Central Universities (No. xzy032020011), the Characteristic Development in Central Universities (No. PY3A077), the Postdoctoral Research Foundation of China under Grant No. 2018M631140, and the Youth program of National Natural Science Foundation of China under Grant No. 12002252.

\section{References}

[1] A. L. Hodgkin and A. F. Huxley, "A quantitative description of membrane current and its application to conduction and excitation in nerve," The Journal of Physiology, vol. 117, no. 4, pp. 500-544, 1952.

[2] J. Guckenheimer and R. A. Oliva, "Chaos in the HodgkinHuxley model," SIAM Journal on Applied Dynamical Systems, vol. 1, no. 1, pp. 105-114, 2002.

[3] R. FitzHugh, "Impulses and physiological states in theoretical models of nerve membrane," Biophysical Journal, vol. 1, no. 6, pp. 445-466, 1961.

[4] J. L. Hindmarsh and R. M. Rose, "A model of neuronal bursting using three coupled first order differential equations," Proceedings of the Royal Society Series B-Biological Sciences, vol. 221, no. 1222, pp. 87-102, 1984.

[5] C. Morris and H. Lecar, "Voltage oscillations in the barnacle giant muscle-FIBER," Biophysical Journal, vol. 35, no. 1, pp. 193-213, 1981.

[6] K. Tsumoto, H. Kitajima, T. Yoshinaga, K. Aihara, and H. Kawakami, "Bifurcations in Morris-Lecar neuron model," Neurocomputing, vol. 69, no. 4-6, pp. 293-316, 2006.

[7] J. M. Gonzalez-Miranda, "Pacemaker dynamics in the full Morris-Lecar model," Communications in Nonlinear Science and Numerical Simulation, vol. 19, no. 9, pp. 3229-3241, 2014.

[8] M. Ciszak and M. Bellesi, "Synaptic plasticity modulates autonomous transitions between waking and sleep states: insights from a Morris-Lecar model," Chaos, vol. 21, no. 4, article 043119, 2011.

[9] X. Hu, C. Liu, L. Liu, J. Ni, and S. Li, "An electronic implementation for Morris-Lecar neuron model," Nonlinear Dynamics, vol. 84, no. 4, pp. 2317-2332, 2016

[10] R. Wang, J. Li, M. Du, J. Lei, and Y. Wu, “Transition of spatiotemporal patterns in neuronal networks with chemical synapses," Communications in Nonlinear Science and Numerical Simulation, vol. 40, pp. 80-88, 2016.

[11] P. Feng, R. Wang, and Y. Wu, In New Trends in Nonlinear DynamicsSpringer International Publishing.

[12] M. Ozer and N. H. Ekmekci, "Effect of channel noise on the time-course of recovery from inactivation of sodium channels," Physics Letters A, vol. 338, no. 2, pp. 150-154, 2005.
[13] L. Wang, S. Liu, J. Zhang, and Y. Zeng, “Temperature-dependent transitions of burst firing patterns in a model pyramidal neuron," Neurophysiology, vol. 44, no. 4, pp. 265-273, 2012.

[14] Q. Wang, Q. Lu, G. Chen, Z. Feng, and L. Duan, "Bifurcation and synchronization of synaptically coupled FHN models with time delay," Chaos Solitons \& Fractals, vol. 39, no. 2, pp. 918925, 2009.

[15] D. Fan and Q. Wang, "Synchronization and bursting transition of the coupled Hindmarsh-Rose systems with asymmetrical time-delays," Science China-Technological Sciences, vol. 60, no. 7, pp. 1019-1031, 2017.

[16] J. T. Porter and K. D. McCarthy, "Hippocampal astrocytes in situ respond to glutamate released from synaptic terminals," Journal of Neuroscience, vol. 16, no. 16, pp. 5073-5081, 1996.

[17] V. Parpura and P. G. Haydon, "Physiological astrocytic calcium levels stimulate glutamate release to modulate adjacent neurons," Proceedings of the National Academy of Sciences of the United States of America, vol. 97, no. 15, pp. 8629-8634, 2000.

[18] G. Arcuino, J. H.-C. Lin, T. Takano et al., "Intercellular calcium signaling mediated by point-source burst release of ATP," Proceedings of the National Academy of Sciences of the United States of America, vol. 99, no. 15, pp. 9840-9845, 2002.

[19] S. Nadkarni and P. Jung, "Spontaneous oscillations of dressed neurons: a new mechanism for epilepsy?," Physical Review Letters, vol. 91, no. 26, 2003.

[20] M. Amiri, N. Hosseinmardi, F. Bahrami, and M. Janahmadi, "Astrocyte- neuron interaction as a mechanism responsible for generation of neural synchrony: a study based on modeling and experiments," Journal of Computational Neuroscience, vol. 34, no. 3, pp. 489-504, 2013.

[21] R. Zhao, S. Zhang, Z. Xu, L. Ju, D. Lu, and G. Yao, "Studying gene expression profile of rat neuron exposed to $1800 \mathrm{MHz}$ radiofrequency electromagnetic fields with cDNA microassay," Toxicology, vol. 235, no. 3, pp. 167-175, 2007.

[22] B. C. Bao, Z. Liu, and J. P. Xu, "Steady periodic memristor oscillator with transient chaotic behaviours," Electronics Letters, vol. 46, no. 3, pp. 228-229, 2010.

[23] Q. Li, H. Zeng, and J. Li, "Hyperchaos in a 4D memristive circuit with infinitely many stable equilibria," Nonlinear Dynamics, vol. 79, no. 4, pp. 2295-2308, 2015.

[24] M. Lv, C. Wang, G. Ren, J. Ma, and X. Song, "Model of electrical activity in a neuron under magnetic flow effect," Nonlinear Dynamics, vol. 85, no. 3, pp. 1479-1490, 2016.

[25] J. Ma, Y. Wang, C. Wang, Y. Xu, and G. Ren, "Mode selection in electrical activities of myocardial cell exposed to electromagnetic radiation," Chaos Solitons \& Fractals, vol. 99, pp. 219-225, 2017.

[26] P. Feng, Y. Wu, and J. Zhang, "A route to chaotic behavior of single neuron exposed to external electromagnetic radiation," Frontiers in Computational Neuroscience, vol. 11, 2017.

[27] P. Feng, Z. Zhang, and Y. Wu, "Stair-like frequency response of single neuron to external electromagnetic radiation and onset of chaotic behaviors," International Journal of Bifurcation and Chaos, vol. 30, no. 9, article 2050131, 2020.

[28] F. Parastesh, K. Rajagopal, A. Karthikeyan, A. Alsaedi, T. Hayat, and V.-T. Pham, "Complex dynamics of a neuron model with discontinuous magnetic induction and exposed to external radiation," Cognitive Neurodynamics, vol. 12, no. 6, pp. 607-614, 2018. 
[29] Z.-X. Yuan, P.-H. Feng, M.-M. Du, and Y. Wu, "Dynamical response of a neuron-astrocyte coupling system under electromagnetic induction and external stimulation," Chinese Physics B, vol. 29, no. 3, article 030504, 2020.

[30] Z. Rostami and S. Jafari, "Defects formation and spiral waves in a network of neurons in presence of electromagnetic induction," Cognitive Neurodynamics, vol. 12, no. 2, pp. 235-254, 2018.

[31] C. Tian, L. Cao, H. Bi, K. Xu, and Z. Liu, "Chimera states in neuronal networks with time delay and electromagnetic induction," Nonlinear Dynamics, vol. 93, no. 3, pp. 1695-1704, 2018.

[32] M. Amiri, G. Montaseri, and F. Bahrami, "On the role of astrocytes in synchronization of two coupled neurons: a mathematical perspective," Biological Cybernetics, vol. 105, no. 2, pp. 153-166, 2011.

[33] M. Bikson, P. J. Hahn, J. E. Fox, and J. G. R. Jefferys, “Depolarization block of neurons during maintenance of electrographic seizures," Journal of Neurophysiology, vol. 90, no. 4, pp. 24022408, 2003. 